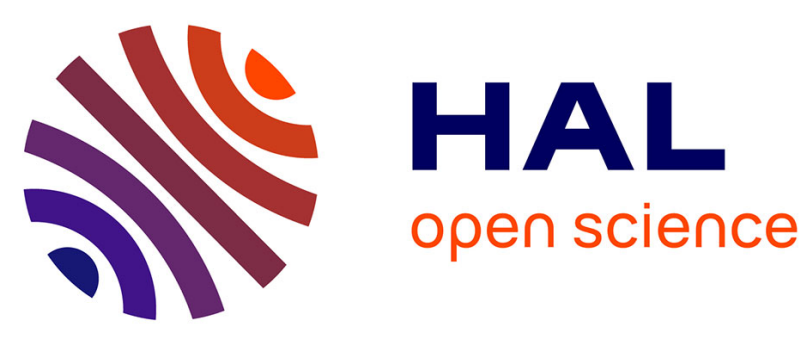

\title{
Interacting cobweb markets
}

\author{
Roberto Dieci, Frank Westerhoff
}

\section{To cite this version:}

Roberto Dieci, Frank Westerhoff. Interacting cobweb markets. Journal of Economic Behavior and

Organization, 2010, 75 (3), pp.461. 10.1016/j.jebo.2010.05.004 . hal-00849411

\section{HAL Id: hal-00849411 \\ https://hal.science/hal-00849411}

Submitted on 31 Jul 2013

HAL is a multi-disciplinary open access archive for the deposit and dissemination of scientific research documents, whether they are published or not. The documents may come from teaching and research institutions in France or abroad, or from public or private research centers.
L'archive ouverte pluridisciplinaire HAL, est destinée au dépôt et à la diffusion de documents scientifiques de niveau recherche, publiés ou non, émanant des établissements d'enseignement et de recherche français ou étrangers, des laboratoires publics ou privés. 


\section{Accepted Manuscript}

Title: Interacting cobweb markets

Authors: Roberto Dieci, Frank Westerhoff

PII:

DOI:

S0167-2681(10)00086-7

Reference: doi:10.1016/j.jebo.2010.05.004

JEBO 2539

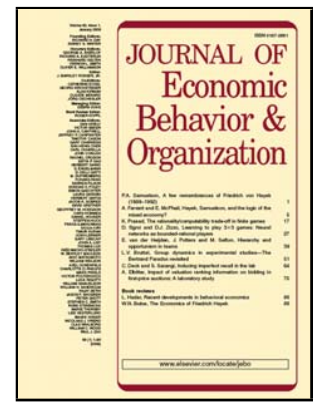

To appear in: Journal of Economic Behavior \& Organization

Received date: $\quad 15-11-2007$

Revised date: $\quad 28-4-2010$

Accepted date: $\quad 11-5-2010$

Please cite this article as: Dieci, R., Westerhoff, F., Interacting cobweb markets, Journal of Economic Behavior and Organization (2008), doi:10.1016/j.jebo.2010.05.004

This is a PDF file of an unedited manuscript that has been accepted for publication. As a service to our customers we are providing this early version of the manuscript. The manuscript will undergo copyediting, typesetting, and review of the resulting proof before it is published in its final form. Please note that during the production process errors may be discovered which could affect the content, and all legal disclaimers that apply to the journal pertain. 


\title{
Interacting cobweb markets*
}

\author{
Roberto Dieci ${ }^{\dagger}$ \\ Department of Mathematics for Economics and Social Sciences \\ University of Bologna \\ Viale Q. Filopanti, 5 \\ I-40126 Bologna, Italy \\ phone: +390541434140 \\ fax: +390541434120 \\ email: roberto.dieci@unibo.it \\ Frank Westerhoff \\ Department of Economics \\ University of Bamberg \\ Feldkirchenstrasse 21 \\ D-49069 Bamberg, Germany \\ phone: +49 (0) 9518632634 \\ fax: +49 (0) 9518635635 \\ email: frank.westerhoff@uni-bamberg.de
}

\footnotetext{
*An early version of this paper was presented at Workshop NED 05 (Nonlinear Economic Dynamics), University of Urbino, July 28-30, 2005. A modified version has been presented at the Seminar 'The Relevance of Network and Complex System Theory for Technological Search and Diffusion', University of Bologna, Faculty of Economics Rimini, June 13-14, 2008. We are grateful to the participants for helpful comments and suggestions. We also thank two anonymous referees for many constructive remarks. The usual disclaimer applies.

${ }^{\dagger}$ Corresponding author. Address: Department of Mathematics for Economics and Social Sciences, University of Bologna, Viale Q. Filopanti 5, I-40126 Bologna, Italy. Phone: +39 0541 434140, Fax: +39 0541434120.
} 


\title{
Interacting cobweb markets
}

\begin{abstract}
We enrich the classical cobweb framework by allowing producers to enter different markets. The market entry decision is repeated every period and depends on the markets' historical profit differentials. As a result, the number of producers in a market and thus also a market's total supply vary over time. Analytical and numerical investigations of our four-dimensional nonlinear model indicate that interacting cobweb markets may contribute to the strong cyclical price motion observed in many commodity markets. We furthermore find that endogenous dynamics may either set in via a Flip or a Neimark-Sacker bifurcation. Interestingly, the latter scenario is prevalent if producers are sufficiently risk averse.
\end{abstract}

Keywords: price fluctuations, market interactions, risk aversion, nonlinear dynamics, bifurcation analysis

JEL classification: C62, E32, Q11

\section{Introduction}

Cobweb models allow the investigation of the price dynamics of a nonstorable good that takes one time unit to produce. Due to the production lag, suppliers must form price expectations one time period ahead. Such a setup is typical for agricultural markets. Consider, for instance, the cultivation of crops. The growing season guarantees a finite lag between the time the production decision is made and the time the crop is ready for sale. The farmer's decision about how much should be produced is usually based on current and past experience. Within the classical cobweb model of Ezekiel (1938), producers simply form naïve expectations. This early model has obtained certain empirical support since it provides an explanation - at least in principle - for the cyclical tendencies observed in many commodity markets. There is also evidence that farmers indeed rely on simple strategies to predict prices (Baak 1999, Chavas 2000). Such behavior has also been detected in laboratory cobweb experiments (Hommes et al. 2007, Sonnemans et al. 2004). However, due to the assumption of linear demand and supply, the range of long-run outcomes that the classical linear cobweb model is able to produce is restricted - in practice - to either damped or exploding oscillations around the equilibrium price, which is why it has never been considered as a very useful tool.

A renewed interest in cobweb models has emerged in the last twenty years, triggered by the growing popularity of nonlinear dynamics as a tool of economic analysis, and the classical linear cobweb model has been extended in several directions. Exploiting nonlinearities in demand and supply, Chiarella (1988), Day (1994), Hommes (1994, 1998) analytically show the possibility of 
chaotic price dynamics for producers' different adaptive expectation schemes. In the seminal work of Brock and Hommes (1997), the demand and supply curves are linear, but producers may switch between different expectation formation rules. Depending on publicly available fitness measures, producers opt for either free naïve or costly rational expectations. The choice is rational in the sense that forecasting rules with a high level of fitness are preferred. The model does not only have the potential to produce complex price dynamics - it also suggests that irregular dynamics may be part of a fully rational notion of equilibrium. Interesting related approaches include, for instance, Goeree and Hommes (2000), Branch (2002), Chiarella and He (2003), Onozaki et al. (2003) and Lasselle et al. (2005). Further nonlinear extensions assume boundedly rational heterogeneous producers and explicitly consider the role of risk aversion and time-varying second moment beliefs (Boussard 1996, Chiarella et al. 2006).

Finally, a number of authors have extended the classical framework to the case of interdependent cobweb economies for substitutes or complements goods, "linked" from the demand side (Currie and Kubin 1995, Hommes and van Eekelen 1996, Yousefi et al. 2000). They have shown how small interdependencies can dramatically affect the dynamics - with respect to the case of independent economies - and have discussed the implications for the relevance of partial analysis in economics.

Also the present paper is devoted to exploring market interdependencies within the cobweb framework, though in this case market interactions are considered from the supply side. The goal of the present paper is to take into account that suppliers produce different goods. For instance, when a farmer decides to reduce his production of rye, he may alternatively expand his production of wheat. To make matters as simple as possible, we consider within our model a situation in which producers may choose between one of two markets. The producers' choice, which depends on how profitable the two markets have been in the recent past, is updated over time. The market which has been more successful for the producers will consequently be selected by more producers than its counterpart. Since the number of producers in a market varies over time, the total supply is nonlinear. Nonlinearity is thus brought into the model not directly, but emerges endogenously by allowing suppliers to switch between 'linear' cobweb markets.

Using analytical and numerical tools we find that our model has the potential to produce long-run price fluctuations, and even complex dynamics. Besides the parameters which govern the slopes of demand and supply schedules, it turns out that the 'switching' parameter, i.e. the agents' sensitivity to past relative profit opportunities, is particularly important for bifurcation analysis. What is remarkable is that market interactions may create endogenous dynamics even for parameter combinations for which both isolated markets would be globally asymptotically stable. In a stylized way, the dynamics evolves as follows. Suppose that some producers switch from the less to the more profitable market. In the less profitable market, the total supply decreases and the price increases. In the other market, the opposite occurs: the total supply increases and the price decreases. Since the profit differential is likely to reverse, some producers stream back to the other market. This pattern may repeat itself in an intricate way.

A further important feature of the model is its ability to generate endogenous fluctuations in different ways - by producing different kinds of local bifurcations - when steady state stability is lost 
due to changes of a key parameter. Such possibly different outcomes may be set in relation with the impact of risk and risk aversion on output decisions. Namely, under sufficiently low risk aversion, or risk perception, of the producers, the emerging scenario is that of a Flip bifurcation, where the system departs from its steady state and prices jump above and below their equilibrium levels, converging to a cyclical orbit of period two. This outcome may be regarded as analogous to the unstable behavior of the traditional linear cobweb. High risk aversion, or strong risk perception, are associated, instead, with the possibility of a Neimark-Sacker bifurcation. This represents a quite new scenario, characterized by more intricate fluctuations of prices. In the presence of demand and (individual) supply schedules of linear type, such an outcome appears therefore as the combined effect of producers' risk aversion and their tendency to switch between markets, depending on relative profit opportunities. Interactions between cobweb markets may thus add to the cyclical component of commodity prices. The model may also be relevant from a policy perspective. Policy makers who intervene in one market should pay great attention to such mechanisms of interaction, in order to anticipate to what extent other markets will be influenced, too. Stabilization schemes have to be planned carefully.

The remainder of our paper is organized as follows. In Section 2, we present a model with two interacting cobweb markets. In Section 3, we reduce the model to a 4-dimensional discrete-time nonlinear dynamical system. We also add some remarks about the impact of our assumptions on the dynamical structure of the model. Analytical results about the steady state of the model are then derived in Section 3.1, whereas its local stability properties are explored, for the case of symmetric markets, in Section 3.2. In Section 4 we present and discuss some simulation results. In particular, Section 4.1 performs a bifurcation analysis, with respect to various parameters, for the reference case of symmetric markets. Section 4.2 introduces asymmetries between markets, and explores their effect on the local stability properties and the global behavior. Section 4.3 performs numerical experiments on some simple generalizations of the model. In Section 5, we summarize our main findings and point out some avenues for future research. The Appendices provide a discussion of the decision problem of the single producer (A1) as well as mathematical details about the steady state (A2) and its local asymptotic stability conditions (A3).

\section{The model}

Traditional cobweb models describe a dynamic price adjustment process on a competitive market for a single non storable good with a supply response lag. We seek to extend this framework by considering two interacting markets, called markets $X$ and $Z$. The interaction arises because producers are able to select the market they want to enter. The market entry decision is repeated at the beginning of each period and depends on the markets' most recent profit differential. For instance, if market $X$ was more profitable than market $Z$, it attracts a higher number of producers. Since the total supply in a market is not constant over time, the model is nonlinear. For simplicity, we specify all other model parts as in the classical cobweb literature.

Let us now formalize the model. We consider a fixed number $N$ of producers. The fractions 
of producers entering markets $X$ and $Z$ at time step $t$ are denoted as $W_{X, t}$ and $W_{Z, t}=1-W_{X, t}$, respectively. An individual producer either supplies quantity $S_{X, t}$ or $S_{Z, t}$. Hence, the total supply in the two markets is $N W_{X, t} S_{X, t}, N W_{Z, t} S_{Z, t}$. Market clearing occurs in every period, implying that

$$
D_{X, t}=N W_{X, t} S_{X, t}, \quad D_{Z, t}=N W_{Z, t} S_{Z, t}
$$

where $D_{X, t}$ and $D_{Z, t}$ denote the demand for goods $X$ and $Z$, respectively.

Consumer demand depends negatively upon the current market price $P_{t}$. Using linear demand curves, demand for goods $X$ and $Z$ may be expressed as

$$
D_{X, t}=\left(a_{X}-P_{X, t}\right) / b_{X}, \quad D_{Z, t}=\left(a_{Z}-P_{Z, t}\right) / b_{Z}
$$

where $a_{X}, a_{Z}, b_{X}, b_{Z}>0$.

The producers' supply depends positively on the expected price. Using linear supply curves and assuming that producers have naïve expectations, $E_{t-1}\left[P_{t}\right]=P_{t-1}$, the supply of a single producer is either

$$
S_{X, t}=\frac{P_{X, t-1}-c_{X}}{d_{X}}
$$

or

$$
S_{Z, t}=\frac{P_{Z, t-1}-c_{Z}}{d_{Z}}
$$

respectively, where $c_{X}, c_{Z} \geq 0, d_{X}, d_{Z}>0$. In the following we assume $a_{X}>c_{X}, a_{Z}>c_{Z} \cdot{ }^{1}$ Note that naïve expectations entail a supply response lag, i.e. the supply in period $t$ depends on the realized price in period $t-1$. The market clearing conditions (1) yield the laws of motion of the two prices

$$
\begin{aligned}
& P_{X, t}=a_{X}-\frac{b_{X}}{d_{X}} N W_{X, t}\left(P_{X, t-1}-c_{X}\right) \\
& P_{Z, t}=a_{Z}-\frac{b_{Z}}{d_{Z}} N W_{Z, t}\left(P_{Z, t-1}-c_{Z}\right) .
\end{aligned}
$$

It is useful to remember what happens if the producers permanently split evenly across the market, i.e. $W_{X, t}=W_{Z, t}=\bar{W}=0.5$. Then the evolution of prices is driven by two independent first-order linear difference equations. The unique fixed point, e.g. of market $X$, is

$$
\bar{P}_{X}=\frac{a_{X} d_{X}+0.5 N b_{X} c_{X}}{d_{X}+0.5 N b_{X}}
$$

and it is globally asymptotically stable if

$$
\left|\frac{N b_{X}}{2 d_{X}}\right|<1 .
$$

Stability of the steady state requires a certain relation between the slopes of the demand and supply

\footnotetext{
${ }^{1}$ Note that such a minimum requirement represents a necessary condition for demand $D_{X, t}$ and supply $S_{X, t}$ (resp. $D_{Z, t}$ and $\left.S_{Z, t}\right)$ to be strictly positive for any $t$.
} 
curves $^{2}$. If (8) is true, then the law of motion (5) of the system under fixed fractions generates temporary up and down price oscillations around the long-run equilibrium price (7) (the same holds for the law of motion (6) of market $Z$ ).

However, our goal is to study endogenous interactions between markets $X$ and $Z$, which result from time varying fractions, depending on the markets' past relative profitability. At the beginning of each period, producers decide which market they will enter. The producers are boundedly rational in the sense that they tend to select the market which would have been more profitable for them in the last period. We assume that the profitability of the two markets in period $t$ is identified by the realized profits $\pi_{X, t}$ and $\pi_{Z, t}$, defined as follows

$$
\pi_{X, t}=P_{X, t} S_{X, t}-C_{X}\left(S_{X, t}\right), \pi_{Z, t}=P_{Z, t} S_{Z, t}-C_{Z}\left(S_{Z, t}\right)
$$

where $C_{X}\left(S_{X}\right)$ and $C_{Z}\left(S_{Z}\right)$ are the cost functions of goods $X$ and $Z$, respectively. Consistent with the assumption of linear supply curves, we will assume that the producers face quadratic cost functions of the type (we omit subscripts) $C(S)=c S+e S^{2}$. Appendix A1 discusses in detail the relationship between the parameters of the supply curves and those of the cost functions, assuming expected utility maximizing risk-averse producers. ${ }^{3}$ Here we simply note that the case of risk-averse producers is characterized by $d>2 e$, while the extreme case $d \rightarrow 2 e$ corresponds to risk neutrality. ${ }^{4}$

Assuming a high number of producers, fractions $W_{X, t}$ and $W_{Z, t}$ can be determined via a discrete choice model (see, e.g. Brock and Hommes 1997, 1998). The fractions of producers selecting market $X$ and $Z$ in period $t$ are given, respectively, by

$$
\begin{aligned}
W_{X, t} & =\frac{\exp \left(f \pi_{X, t-1}\right)}{\exp \left(f \pi_{X, t-1}\right)+\exp \left(f \pi_{Z, t-1}\right)}, \\
W_{Z, t} & =\frac{\exp \left(f \pi_{Z, t-1}\right)}{\exp \left(f \pi_{X, t-1}\right)+\exp \left(f \pi_{Z, t-1}\right)}=1-W_{X, t},
\end{aligned}
$$

where the realized profits can be expressed as

$$
\pi_{X, t-1}=\left(P_{X, t-1}-c_{X}\right) S_{X, t-1}-e_{X} S_{X, t-1}^{2}, \quad \pi_{Z, t-1}=\left(P_{Z, t-1}-c_{Z}\right) S_{Z, t-1}-e_{Z} S_{Z, t-1}^{2},
$$

with $e_{X}, e_{Z}>0$. Note that the higher the profitability of a market, the more producers will enter that market. The parameter $f>0$ is the intensity of choice and measures how sensitive the mass of producers is to selecting the most profitable market. An increase in $f$ may be interpreted as

\footnotetext{
${ }^{2}$ Note that in the case of equal proportions, the quantities $-1 / b_{X}$ and $N /\left(2 d_{X}\right)$ represent the slopes of the demand curve and the aggregated supply curve, respectively, so that (8) turns out to be the familiar condition for stability of the classical cobweb model.

${ }^{3}$ Given the assumption of risk averse producers, a perhaps more reasonable measure of profitability should consider also a risk-adjustment term. However, for the sake of simplicity we follow Brock and Hommes (1997, 1998), and assume that agents only take realized profits into account. We leave the case of risk adjusted profits for future research. On the other hand, as also noticed by Brock and Hommes (1998), from a practical viewpoint realized profits may be what agents care most about.

${ }^{4}$ Dieci and Westerhoff (2009) explicitly focus on the case in which agents are risk neutral, under general demand and cost functions. In this framework it is possible to extract some additional results, in particular when the markets are asymmetric.
} 
an increase in the (bounded) rationality of the agents. For $f=0$, the agents do not observe any profit differentials between the two markets. As a result, $W_{X, t}=W_{Z, t}=\bar{W}=0.5$ for any $t$ and we are back to the fixed fraction model. The case of independent markets can then be recovered as a particular case for $f=0$. On the other hand, the higher $f$, the more producers enter the more profitable market in the following period. In the extreme case $f \rightarrow \infty$, all producers switch to the market with the higher profitability in the preceding period.

\section{The dynamical system}

Note that by substituting (9)-(10) into (5)-(6), the model is expressed by a system of two nonlinear second-order difference equations in the prices, in that $\pi_{X, t-1}\left(\pi_{Z, t-1}\right)$ turns out to depend both on $P_{X, t-1}$ and on $P_{X, t-2}\left(P_{Z, t-1}\right.$ and $\left.P_{Z, t-2}\right)$. The system can be rewritten as a 4-dimensional dynamical system in the dynamic variables $P_{X}, P_{Z}, S_{X}, S_{Z}$. In the following analysis, we normalize the total number of producers to $N=1 .{ }^{5}$ Moreover, in order to simplify the dynamic analysis, we define the difference of fractions ${ }^{6} \Omega_{t}:=W_{X, t}-W_{Z, t}$, which can be rewritten as

$$
\Omega_{t}=\tanh \left[\frac{f}{2}\left(\pi_{X, t-1}-\pi_{Z, t-1}\right)\right],
$$

with $-1<\Omega_{t}<1$, where $\Omega_{t} \rightarrow 1$ corresponds to $W_{X, t} \rightarrow 1$ and $\Omega_{t} \rightarrow-1$ corresponds to $W_{Z, t} \rightarrow 1$. Note also that from $W_{X, t}+W_{Z, t}=1$ one immediately obtains $W_{X, t}=\left(1+\Omega_{t}\right) / 2, W_{Z, t}=\left(1-\Omega_{t}\right) / 2$. The resulting $4-D$ dynamical system is thus the following

$$
\begin{gathered}
P_{X, t}=\frac{a_{X} d_{X}-g_{X}\left(1+\Omega_{t}\right)\left(P_{X, t-1}-c_{X}\right)}{d_{X}}, \\
P_{Z, t}=\frac{a_{Z} d_{Z}-g_{Z}\left(1-\Omega_{t}\right)\left(P_{Z, t-1}-c_{Z}\right)}{d_{Z}}, \\
S_{X, t}=\frac{P_{X, t-1}-c_{X}}{d_{X}}, \\
S_{Z, t}=\frac{P_{Z, t-1}-c_{Z}}{d_{Z}},
\end{gathered}
$$

where $g_{X}:=b_{X} / 2, g_{Z}:=b_{Z} / 2 . \Omega_{t}$ is itself a function of the state variables at time $t-1$ :

$$
\Omega_{t}=\tanh \left\{\frac{f}{2}\left[\left(P_{X, t-1}-c_{X}\right) S_{X, t-1}-e_{X} S_{X, t-1}^{2}-\left(P_{Z, t-1}-c_{Z}\right) S_{Z, t-1}+e_{Z} S_{Z, t-1}^{2}\right]\right\} .
$$

Before we start the dynamic analysis, we add a few remarks about certain simplifying assumptions behind our setup, and briefly discuss their impact on the dynamic structure of model (11)-(14).

- The model incorporates market interactions only from the supply side and neglects demand

\footnotetext{
${ }^{5}$ Which is equivalent to rescaling the parameters $b_{X}$ and $b_{Z}$ in (5) and (6), respectively.

${ }^{6}$ The same change of variable is used by Brock and Hommes $(1997,1998)$.
} 
side effects. As a result, demand in a market is supposed to be a function of its own good's price solely. Introduction of cross-dependencies would complicate dynamic equations (11) and (12), thus rendering analytical results more difficult. Our assumption represents, of course, a rather strong premise. ${ }^{7}$ We consider this as a working assumption, that enables an initial understanding of the effect of interactions between cobweb markets, in comparison with the case of independent markets. The results we obtain are also robust enough, as can be seen from Section 4.3, where we explore numerically the case of more general demand functions, with positive or negative cross-elasticities (substitutes or complements goods).

- We assume that profitability of the two markets in a certain period is identified by realized profits. This raises two caveats. ${ }^{8}$ First, it naturally requires every supplier to be informed about profitability in both markets, which may be regarded as a strong assumption. However, the existence of publicly available fitness (profitability) measures for each 'strategy' represents a quite common assumption in this branch of literature, that may be justified by assuming that agents talk to each other about their activities. Alternatively, realized profits may be inferred from observed prices (note that e.g. $\pi_{Z, t-1}$ is uniquely determined by $P_{Z, t-1}$ and $P_{Z, t-2}$ ). Second, our simplified setup abstracts from economies of scale and scope that might play a role, in particular, for agricultural markets and commodities. Note that the weights $W_{X, t}$ and $W_{Z, t}$ could also be interpreted as fractions of outputs $X$ and $Z$, respectively, produced by one representative agent. If we adopt this view, the parallel production of - say - rye and wheat, should be modelled as a combined production process, and thus the cost functions, as well as realized profits of the two goods, would no longer be independent.

- For the sake of simplicity, we adopt the assumption of naïve expectations. A more general starting point would be the cobweb model with an adaptive price expectation scheme à la Nerlove (1958). This would introduce, however, two additional dynamic variables into the model, namely, the expected prices of goods $X$ and $Z$, without substantially modifying the qualitative nature of the results.

The following subsections explore the steady state properties of the model.

\subsection{Steady state}

Let us now derive the stationary states of the model. The steady states of the system are obtained by setting $\left(P_{X, t}, P_{Z, t}, S_{X, t}, S_{Z, t}\right)=\left(P_{X, t-1}, P_{Z, t-1}, S_{X, t-1}, S_{Z, t-1}\right)=\left(\bar{P}_{X}, \bar{P}_{Z}, \bar{S}_{X}, \bar{S}_{Z}\right)$ in the dynamical system (11)-(14). The system admits a unique steady state, as stated in the following

\footnotetext{
${ }^{7}$ With this assumption, a possible interpretation of the model is that it depicts a situation where markets are geographically separated because of prohibitively high transportation costs, i.e. consumers in one market cannot go to the other market. We thank an anonymous referee for having suggested this interpretation.

${ }^{8} \mathrm{We}$ are grateful to an anonymous referee for having raised such remarks.
} 
Proposition 1 There exists a unique steady state of the nonlinear dynamical system (11)-(14). The steady state distribution of producers across markets, $\bar{\Omega}$, is implicitly defined by the equation

$$
\Omega=\tanh \left\{\frac{f}{2}\left[\frac{\left(d_{X}-e_{X}\right)\left(a_{X}-c_{X}\right)^{2}}{\left(d_{X}+g_{X}(1+\Omega)\right)^{2}}-\frac{\left(d_{Z}-e_{Z}\right)\left(a_{Z}-c_{Z}\right)^{2}}{\left(d_{Z}+g_{Z}(1-\Omega)\right)^{2}}\right]\right\}
$$

while the stationary prices and supply levels are given by

$$
\begin{gathered}
\bar{P}_{X}=\frac{a_{X} d_{X}+g_{X}(1+\bar{\Omega}) c_{X}}{d_{X}+g_{X}(1+\bar{\Omega})}, \quad \bar{P}_{Z}=\frac{a_{Z} d_{Z}+g_{Z}(1-\bar{\Omega}) c_{Z}}{d_{Z}+g_{Z}(1-\bar{\Omega})}, \\
\bar{S}_{X}=\frac{\bar{P}_{X}-c_{X}}{d_{X}}=\frac{a_{X}-c_{X}}{d_{X}+g_{X}(1+\bar{\Omega})}, \quad \bar{S}_{Z}=\frac{\bar{P}_{Z}-c_{Z}}{d_{Z}}=\frac{a_{Z}-c_{Z}}{d_{Z}+g_{Z}(1-\bar{\Omega})} .
\end{gathered}
$$

The proof is contained in Appendix A2. We add here a few important remarks, that follow from Proposition 1. Note first that the steady state cannot be computed explicitly in general and that the stationary levels of the state variables depend on the intensity of choice $f$. Note also that the steady-state profits $\bar{\pi}_{X}=\left(\bar{P}_{X}-c_{X}\right) \bar{S}_{X}-e_{X} \bar{S}_{X}^{2}, \bar{\pi}_{Z}=\left(\bar{P}_{Z}-c_{Z}\right) \bar{S}_{Z}-e_{Z} \bar{S}_{Z}^{2}$ can be rewritten as

$$
\begin{aligned}
& \bar{\pi}_{X}=\left(d_{X}-e_{X}\right) \bar{S}_{X}^{2}=\frac{\left(d_{X}-e_{X}\right)\left(a_{X}-c_{X}\right)^{2}}{\left(d_{X}+g_{X}(1+\bar{\Omega})\right)^{2}}, \\
& \bar{\pi}_{Z}=\left(d_{Z}-e_{Z}\right) \bar{S}_{Z}^{2}=\frac{\left(d_{Z}-e_{Z}\right)\left(a_{Z}-c_{Z}\right)^{2}}{\left(d_{Z}+g_{Z}(1-\bar{\Omega})\right)^{2}},
\end{aligned}
$$

which are part of the right-hand side of (16). Of course, the case of independent markets, $f=0$, results in $\bar{\Omega}=0$, and steady states of the two markets are as in the traditional cobweb. We denote by $\bar{P}_{X}^{0}, \bar{P}_{Z}^{0}, \bar{S}_{X}^{0}, \bar{S}_{Z}^{0}, \bar{\pi}_{X}^{0}, \bar{\pi}_{Z}^{0}$, respectively, quantities (17)-(20) computed for $\bar{\Omega}=0$. They represent steady state prices, quantities, and profits of the two markets considered in isolation. We can then compare the steady state of the model with interacting markets with that of the case of independent markets. If we denote by $Q(\Omega)$ the righ-hand side of (16), it follows from the proof of Proposition 1 that $\bar{\Omega}>0$ (resp. $\bar{\Omega}<0$ ) if and only if $Q(0)>0$ (resp. $Q(0)<0$ ), that is to say, if and only if $\bar{\pi}_{X}^{0}>\bar{\pi}_{Z}^{0}$ (resp. $\bar{\pi}_{X}^{0}<\bar{\pi}_{Z}^{0}$ ). Put differently, market $X$ (market $Z$ ) attracts a larger fraction of producers at the steady state if and only if its steady state profit in isolation is higher. For $\bar{\pi}_{X}^{0}>\bar{\pi}_{Z}^{0}$ we also easily obtain $\bar{P}_{X}<\bar{P}_{X}^{0}, \bar{S}_{X}<\bar{S}_{X}^{0}, \bar{\pi}_{X}<\bar{\pi}_{X}^{0}$, and vice versa, for $\bar{\pi}_{X}^{0}<\bar{\pi}_{Z}^{0}$ we obtain $\bar{P}_{X}>\bar{P}_{X}^{0}, \bar{S}_{X}>\bar{S}_{X}^{0}, \bar{\pi}_{X}>\bar{\pi}_{X}^{0}$.

As far as local stability properties of the steady state are concerned, they cannot be easily derived for the general case of asymmetric markets. Analytical study is possible, however, in the particular case where markets are symmetric, i.e. their parameters are identical. This case is explored in the next section. On the other hand, one can easily check by means of numerical experiments (performed in Section 4.2) that the dependence of the local asymptotic stability properties on the key parameters in the general case is qualitatively much the same as in the case of symmetric markets. 


\subsection{Symmetric markets}

In this section we focus on the case where the two markets are symmetric, i.e. their parameters are identical, $a_{X}=a_{Z}:=a, g_{X}=g_{Z}:=g, c_{X}=c_{Z}:=c, d_{X}=d_{Z}:=d, e_{X}=e_{Z}:=e$. Such an assumption is not crucial for our results. The symmetric case just represents an analytically tractable 'reference' case $^{9}$, and the local stability results that we obtain in this case (in particular the existence of two types of bifurcations) admit a straightforward economic interpretation in terms of the parameters. In addition, this case proves very useful in order to compare the dynamics under the assumption of interacting markets $(f>0)$ with the dynamics in the case of independent markets $(f=0)$. The results we obtain in the symmetric case are also quite robust and will be confirmed (though via numerical simulation only) for the general model (see Section 4.2).

Symmetric markets obviously possess the same equilibrium prices. Since the profitability of the two markets is equal in equilibrium, the producers are evenly divided between the two markets, i.e. $\Omega=0$. The steady state then turns out to be defined by $P_{X}=P_{Z}=\bar{P}, S_{X}=S_{Z}=\bar{S}$, where

$$
\bar{P}:=\frac{a d+c g}{d+g}, \quad \bar{S}:=\frac{\bar{P}-c}{d}=\frac{a-c}{d+g} .
$$

Note that the steady state coordinates are independent of parameter $f$ in this particular case. The same parameter instead plays an important role in the local asymptotic stability of the steady state. Before discussing such conditions, in order to appreciate the effect of $f$ on the stability, we remark once again that under the case $f=0$ (independent symmetric markets) the equilibra of the two markets are stable if the slopes of demand and supply satisfy

$$
0<g_{X} / d_{X}=g_{Z} / d_{Z}=g / d<1
$$

As we shall prove, in the case of interacting markets, i.e. for $f>0$, we obtain more restrictive conditions with regard to the slopes of the demand and supply curves. The stability conditions and the possible local bifurcations are summarized in the following

Proposition 2 The steady state in the case of symmetric markets is locally asymptotically stable in the region of the space of parameters where the following inequality is satisfied

$$
\frac{g}{d}<\min \left\{u_{F}, u_{N S}\right\}
$$

where $u_{F}:=1 /\left(1+2 e f \bar{S}^{2}\right), u_{N S}:=1 /\left[f(d-2 e) \bar{S}^{2}\right]$, and $\bar{S}=(a-c) /(d+g)$. In addition, if $g / d<u_{F}<u_{N S}$ and one of the parameters is varied so that $g / d$ becomes larger than $u_{F}$, then a Flip bifurcation takes place. If $g / d<u_{N S}<u_{F}$ and one of the parameters is varied so that $g / d$ becomes larger than $u_{N S}$, then a Neimark-Sacker bifurcation takes place.

The proof is left to Appendix A3. Since $\min \left\{u_{F}, u_{N S}\right\}<1$ for $f>0$, it follows that the

\footnotetext{
${ }^{9}$ If the symmetry assumption were to be interpreted in a strict sense, this would imply cost functions identity and thus, essentially, the modelling of a single good. In Section 4.2, among other experiments on the dynamics of the asymmetric model, we also remove cost identity via bifurcation analysis.
} 
conditions on the slopes of the demand and supply curves needed to ensure local stability are stricter than in the case where producers do not switch between markets. That is to say, if agents are allowed to select the most profitable market, this has a 'destabilizing' effect on the equilibrium prices. Note also that the higher $f$, the smaller the region of the space of parameters where condition (22) is fulfilled ${ }^{10}$, while for $f \rightarrow 0$ the same condition is obviously reduced to $g / d<1$ (and stability can only be lost via Flip bifurcation). In our model with linear demand and supply, therefore, the existence of supply-side interactions between markets, whose 'strength' depends on $f$, brings about the possibility of complex eigenvalues and Neimark-Sacker bifurcation. A further important remark concerns the relationship between the bifurcation structure and the risk, or the risk aversion of the producers. As shown in Appendix A1, if producers are risk-neutral or they perceive zero risk $(d=2 e)$, then $u_{N S} \rightarrow \infty$ and the Neimark-Sacker bifurcation scenario is absent: in this extreme case stability can only be lost via Flip bifurcation. This fact deserves a further comment. As implied by the derivation of supply curves in Appendix A1, the quantity $(d-2 e)$ (inversely related to $u_{N S}$ ) can be interpreted as a kind of 'risk premium' required by producers, positively related to their risk aversion coefficient and perceived risk. Our local results then suggest that when risk aversion / perception is sufficiently small, the steady state will typically lose stability via a Flip bifurcation, with market prices jumping up and down alternately around their equilibrium levels, while the Neimark-Sacker bifurcation scenario, characterized by fluctuations of different nature, will prevail when risk aversion / perception is large enough. ${ }^{11}$ This is further illustrated numerically in the next section. The range of possible dynamic outcomes is then substantially enriched by the explicit introduction of market interactions, and turns out to be qualitatively related to producers' risk attitudes and risk beliefs.

\section{Computational analysis}

Now we are ready to numerically explore the global $^{12}$ dynamics of the model. We are especially interested in the question of whether simple interactions between cobweb markets may generate cyclical or more intricate price movements, in understanding the role of the key parameters on the nature of such fluctuations, and in exploring the dynamics of some straightforward generalizations of the model. In order to perform this task, in the next subsection we focus first on the case where the two markets are symmetric. In subsection 4.2 we introduce asymmetries in either demand or supply parameters, and explore their impact on both the local bifurcations of the steady state and the global dynamical behavior of the system. Finally, subsection 4.3 contains numerical simulations on a number of possible extensions of the model.

\footnotetext{
${ }^{10}$ In the sense that, if $f_{2}>f_{1}$, the stability region (22) for fixed $f=f_{2}$ is strictly included in the stability region for $f=f_{1}$.

${ }^{11}$ In fact, this result is a testable implication of our model. For instance, for regional agricultural markets characterized by machinery syndicates that pool the risk of investments in expensive equipment and fixed capital - thus reducing individual risks - we should expect a prevailing Flip bifurcation scenario. For regions characterized by a more scattered structure of farmers we would expect higher risk, i.e., a prevailing Neimark-Sacker bifurcation scenario. We thank an anonymous referee for suggesting this interesting possible application to real world phenomena.

${ }^{12}$ The term "global" refers to those dynamic phenomena that cannot be detected from the analysis of the linearized system in a neighborhood of the steady state.
} 


\subsection{Global dynamics for symmetric markets}

In the case of symmetric markets, the local stability analysis carried out in the previous section has shown that the steady state may become unstable via both Neimark-Sacker bifurcation and Flip bifurcation. Numerical analysis confirms the existence - under different sets of parameters of an attracting closed curve generated by the Neimark-Sacker bifurcation and an attracting orbit of period 2 generated by the Flip bifurcation. The Flip bifurcation scenario is typical in cobweb models and essentially comes from producers' overreaction to price changes. Here we focus mainly on the long-run price fluctuations generated by the Neimark-Sacker bifurcation, which appear to be strictly related to combined effect of market interactions and risk, as discussed in the previous section.

We use the following parameter setting for our simulation analysis: $a=20, b=6, c=2, d=8$, $e=1$. For $f=0.17$, Fig. 1 shows from top to bottom time series for the price in market $X$, the profitability of market $X$, the price in market $Z$, the profitability of market $Z$ and the distribution of producers across markets (remember that $W_{Z, t}=1-W_{X, t}$ ). As can be seen, prices, profits and suppliers' proportions fluctuate in both markets in an intricate way around their long-run equilibrium values.

\section{Figure 1 goes about here}

What is driving the dynamics? Suppose that supply in market $X$ is low in period $t$. As a result, consumers will pay a high price for good $X$. Market $X$ is therefore quite profitable in period $t$. In the next period, producers update their market entry decision. Given the profitability of market $X$, some producers may migrate from market $Z$ to market $X$. A lower supply in market $Z$ will, however, lead to a price increase of good $Z$. Simultaneously, an increasing supply in market $X$ implies that prices will decrease here. Combined, these two effects may reverse the profit differential in favor of market $Z$ again. This pattern may repeat itself, yet in an intricate way.

Parameter $f$, i.e. the agents' sensitivity to profit differentials, plays a crucial role in driving the evolution of prices: higher sensitivity in general increases the amplitude of the fluctuations of prices, profits and distribution of producers across markets. This is revealed, for instance, by the phase space plots represented in Fig. 2 for the cases $f=0.17$ and $f=0.2$, respectively. For each parameter selection considered in Fig. 2, we represent the projection of the attractor in the planes $P_{X}, P_{Z}$ and $P_{X}, S_{X}$, respectively. In particular, the projections in the plane $P_{X}, P_{Z}$ reveal a strong negative correlation between the prices of the two commodities. As already mentioned, in both cases the time evolution of the system is characterized by quasiperiodic motion on an attracting closed curve in the phase space, and the size of the attractor increases as $f$ increases. Quasiperiodic motion means that prices oscillate in a more or less harmonic manner. Note that actual commodity price fluctuations (Borenzstein et al. 1994, Deaton 1999, Cashin et al. 2002) are characterized by a strong cyclical component. One can easily check that - under our base parameter selection - the birth of the attracting curve via Neimark-Sacker bifurcation occurs when $f$ is increased beyond the bifurcation value $f \simeq 0.166 .^{13}$

\footnotetext{
${ }^{13}$ For different constellations of parameters, an attracting orbit of period 2 exists around the unstable steady state,
} 


\section{Figure 2 goes about here}

Further analysis reveals that the model is able to produce even more complex dynamic scenarios. For instance, one may observe the emergence of a strange attractor (which is a sign of chaotic dynamics) from the attracting closed curve, if $f$ is sufficiently large. Moreover, the coexistence of an attracting 2-cycle and a strange attractor can be detected under our base set of parameters, for instance for $f=0.64295$. This case is represented in Fig. 3, which shows trajectories generated by two different initial conditions, both very close to the repelling steady state, but each selected from a different basin of attraction: one from the basin of the strange attractor (first panel), the other from the basin of the attracting 2-cycle (second panel). The (projections of the) two coexisting attractors on plane $P_{X}, P_{Z}$ are represented, respectively, in the third line of panels. In this case, complex behavior is associated to both the asymptotic dynamics on the strange attractor and the complicated structure of the basins of attraction (bottom panel of Fig. 3).

\section{Figure 3 goes about here}

The bifurcation diagrams represented in Fig. 4 give an idea of how the nature of the asymptotic dynamics depends on the key parameters. Since the markets are symmetric, it suffices to concentrate on one market (market $X$ in our case). Fig. 4 contains four bifurcation diagrams for supply parameter $d$ (first panel), demand parameter $b$ (second panel), cost parameter $c$ (third panel), and the intensity of choice (or bounded rationality) parameter $f$ (fourth panel). The starting parameter selection is $a=20, b=6, c=2, d=8, e=1, f=0.17$, which is characterized by quasiperiodic motion (see Fig. 2). Simple visual inspection reveals that the dynamic behavior of the system may change dramatically as the bifurcation parameter varies. A number of aspects deserve our attention. First, in all four panels convergence towards a steady state is possible. For instance, when the degree of (bounded) rationality is decreased, the slope of the demand curve is increased ( $b$ is decreased) or the supply curve becomes more flat ( $d$ is increased) with respect to the base parameter setting, endogenous dynamics dies out and the system settles down on its long term equilibrium. The effect of increasing cost parameter $c$ is qualitatively similar to that of increasing the supply parameter $d$. Second, the range of parameter values that yield endogenous dynamics, especially quasi-periodic behavior, is very wide. Third, in a similar fashion to what occurs with regard to the local asymptotic stability, also from the point of view of the global dynamics, one could say that the system is 'destabilized' when $d$ decreases, or $b$ increases, in the sense that the range of the fluctuations around the unstable steady state becomes wider. Fourth, as $d$ increases or $b$ decreases the steady state price increases as one would expect: however, when the underlying system dynamics is not a steady state, this usual claim is not that clear, though a similar feature seems to hold for the 'average' price.

\section{Figure 4 goes about here}

born via Flip bifurcation. A Flip bifurcation can be observed, for instance, under the following parameter selection: $a=20, b=14, c=2, d=8, e=1$, when $f$ is increased beyond the bifurcation value $f \simeq 0.0496$. Similar to the case of quasiperiodic motion, the fluctuations on the attracting 2-cycle become wider for higher values of $f$. 
The foregoing numerical experiments, together with the local analysis developed in section 3.2, allow us to summarize the key findings for the reference case of symmetric markets, as follows.

- Endogenous, cyclical or more complex fluctuations may arise, uniquely due to the assumed interaction mechanism between linear cobweb markets, for sufficiently high values of the switching parameter. Moreover, such behavior often occurs for a range of demand and supply parameters that would be compatible with stability of both markets considered in isolation. ${ }^{14}$

- When steady state stability is lost, a strong connection exists between the nature of the emerging fluctuations (a 2-cycle vs. more involved dynamic patterns on a closed curve) and the implicitly assumed risk-aversion and risk perception of the producers.

- Outside the stability domain in the space of parameters, the effect of changes of the slopes of the demand and supply curves is consistent, in a sense, with their role in steady state stability of the traditional cobweb. That is, a 'destabilizing effect' with wider fluctuations and even complex behavior emerges when the supply (demand) curve becomes more sloped (flat).

- Increasing levels of sensitivity to profit differentials (parameter $f$ ) makes the system to evolve from convergence to steady state to fluctuations of increasing size, to more complex behaviors.

Such results have been proven for symmetric interacting cobweb markets. In addition, as already discussed in Section 3, the model is based upon a number of simplifying assumptions. In particular, though we allow markets to be linked from the supply side, we keep them separated from the side of demand, by assuming demand schedules that are independent of the price of the alternative good. Our findings do not constitute, therefore, a general proof that connections between cobweb markets necessarily bring about such kind of endogenous dynamics and complex behavior. Our simplified setup has mainly been adopted, however, for the purpose of analytical tractability and easier economic interpretation of the results. As a matter of fact, the key findings summarized above continue to hold, and are sometimes even stronger, when some assumptions are relaxed. Without aiming at completeness, we illustrate this via numerical examples in the next subsections.

\subsection{Breaking the symmetry}

Let us now consider the effect of breaking the symmetry of the interacting markets. The first numerical example investigates the stability conditions of the steady state, and their dependence on parameter $f$ and on the supply parameter $d_{Z}$ of commodity $Z$. Parameter $d_{Z}$ incorporates, as already remarked, both the coefficient $e_{Z}$ of the quadratic cost term and the risk term, so that increasing $d_{Z}$ for fixed $e_{Z}$ means assuming higher risk perception. The two-parameter bifurcation diagram in Fig. 5, with $d_{Z}$ on the horizontal axis and $f$ on the vertical axis, highlights the effect of various combinations of the switching parameter and the supply parameter in market $Z$. The

\footnotetext{
${ }^{14}$ This represents indeed an important point that we have only briefly sketched in Section 3.2. A careful study of the 'destabilizing' impact of supply-side connections between cobweb economies has been performed in Dieci and Westerhoff (2009), under the assumption of profit-maximizing producers and general demand and cost functions.
} 
parameter setting represents a 'perturbation' of the symmetric case of Fig. 2: parameters of market $X$ are as in Fig. 2, i.e. $a_{X}=20, b_{X}=6$ (and $g_{X}:=b_{X} / 2=3$ ), $c_{X}=2, d_{X}=8, e_{X}=1$, whereas for market $Z$ we choose $a_{Z}=18, b_{Z}=4$ (and $g_{Z}:=b_{Z} / 2=2$ ), $c_{Z}=1, e_{Z}=2$. Note first that under the assumed parameter selection, both markets would be 'stable' in the absence of interactions (in particular, steady state in market $Z$ would be stable for the whole range of $d_{Z}$ considered on the horizontal axis, $5<d_{Z}<9$ ). The white area of the parameter plane corresponds to a locally stable steady state, the gray area to an attracting 2-cycle, while other kinds of dynamic behaviour (including quasiperiodic motion on a closed curve) are represented in black. The phase plots in the two smaller panels are projections in the plane $P_{X}, S_{X}$, obtained with parameter combinations outside the stability region, for $d_{Z}=6, f=0.2$ (two-cycle), and $d_{Z}=8, f=0.24$ (closed curve), respectively. The picture then shows that, when $f$ increases, stability is lost via Flip bifurcation only if $d_{Z}$ is small enough. For higher values of $d_{Z}$ (which means stronger risk perceptions or more risk averse agents) the bifurcation mechanism is in fact that of a Neimark-Sacker bifurcation, as can be checked by looking at the phase-space. Similar pictures are easily obtained by varying parameter $d_{X}$, or by changing the levels of the fixed parameters, as we have checked. This suggests that, with regard to the local bifurcation structure, the qualitative results obtained for symmetric markets in section 3.2 are relatively robust.

\section{Figure 5 goes about here}

Let us now consider a parameter range for which the steady state is unstable and the dynamics are characterized by quasiperiodic fluctuations. Again, we allow the slope of the supply curve in market $Z$ (inversely related to parameter $d_{Z}$ ) to vary, with all other parameters remaining unchanged. In the top panels of Fig. 6 we perturb the symmetric case in the base parameter selection of Figs. 1 and 2 (with $f=0.17$ ) by decreasing $d_{Z}$ from 8 to 6 . The top-left panel in Fig. 6 represents the symmetric case, the top-right panel the asymmetric case. We can observe a 'destabilizing' effect, namely wider fluctuations along a more complex attractor than in the symmetric case. This is also revealed by the corresponding price time paths, in the second line of panels. On the contrary, a 'stabilizing' effect (fluctuations of reduced amplitude) is obtained by increasing parameter $d_{Z}$, as one can easily check. The effect of this symmetry breaking on the asymptotic dynamics, for $d_{Z}$ ranging in the interval [6,10], can be appreciated from the bifurcation diagrams in the third line of panels in Fig. 6. A similar destabilizing (stabilizing) effect is obtained by increasing (decreasing) the demand parameter $b_{Z}$, which governs the slope of the demand curve in market $Z$. See the fourth line of panels. Finally, the diagrams on the bottom line show the effect of increasing the cost parameter $c_{Z} \cdot{ }^{15}$ From this exercise we can conclude the following: The effects of changing demand and supply parameters in the asymmetric scenarios are qualitatively similar to what we observe in the symmetric scenarios (compare Fig. 6 with Fig. 4), both with respect to the stability/instability of the steady state and the amplitude of the fluctuations. In addition, when symmetry is broken there is a tendency towards more irregular fluctuations and increasing complexity, as suggested by the top panels in Fig. 6 .

\footnotetext{
${ }^{15}$ This shows, in particular, that assuming different cost functions (which implies that commodities $X$ and $Z$ are in fact two different goods) does not alter qualitatively the dynamics.
} 


\section{Figure 6 goes about here}

\subsection{Further extensions}

The present subsection is devoted to numerically explore a number of straightforward generalizations of the model. A first goal of such numerical experiments is to provide further evidence of the robustness of our findings. A second goal is to suggest how the interaction mechanism designed in this paper might have further interesting implications, when embedded within more elaborated versions of the model. We consider the following extensions: (a) demand schedules depending on both prices; (b) central authority interventions; (c) exogenous noise.

Demand depending on both prices. Assume that equations (2) take the following more general form:

$$
D_{X, t}=\frac{a_{X}-P_{X, t}-h_{X} P_{Z, t}}{b_{X}}, \quad D_{Z, t}=\frac{a_{Z}-P_{Z, t}-h_{Z} P_{X, t}}{b_{Z}},
$$

where parameters $h_{X}$ and $h_{Z}$ are positive if goods $X$ and $Z$ are complements, negative if the goods are substitutes. As a consequence, the dynamic pricing equations (11) and (12) are modified, respectively as follows:

$$
\begin{aligned}
& P_{X, t}=\frac{1}{1-h_{X} h_{Z}}\left[\frac{a_{X} d_{X}-g_{X}\left(1+\Omega_{t}\right)\left(P_{X, t-1}-c_{X}\right)}{d_{X}}-h_{X} \frac{a_{Z} d_{Z}-g_{Z}\left(1-\Omega_{t}\right)\left(P_{Z, t-1}-c_{Z}\right)}{d_{Z}}\right], \\
& P_{Z, t}=\frac{1}{1-h_{X} h_{Z}}\left[\frac{a_{Z} d_{Z}-g_{Z}\left(1-\Omega_{t}\right)\left(P_{Z, t-1}-c_{Z}\right)}{d_{Z}}-h_{Z} \frac{a_{X} d_{X}-g_{X}\left(1+\Omega_{t}\right)\left(P_{X, t-1}-c_{X}\right)}{d_{X}}\right],
\end{aligned}
$$

whereas equations (13), (14), and (15) remain unchanged.

Fig. 7 (top panel) reports the results of a simple numerical experiment carried out in the symmetric case, with the same parameter setting used for Fig. 1 (and Fig. 2, top panels). The bifurcation parameter is $h_{X}=h_{Z}:=h$. The case of our basic model, where $h_{X}=h_{Z}=0$, is exactly in the middle of the diagram. Two remarks seem important. First, the effect of substitute goods is destabilizing (wider fluctuations), while the converse is true for complement goods (reduced size of fluctuations and even stabilization to steady state). The larger the modulus of $h$, which is related to the cross elasticity of demand, the stronger the destabilizing / stabilizing effects. Second, for a certain range of the parameter around $h=0$, the qualitative behavior of the model with demandside interdependencies is similar to that of independent demands. In particular, the emergence of price fluctuations, for sufficiently large $f$, is observed for the case of both substitute and complement goods. This strengthens our findings about the impact of the switching parameter. Similar results can be obtained by repeating this experiment in the asymmetric case.

Policy implications. The effect of central authority interventions may be an important issue within the market model developed in this paper. A first obvious remark concerns the difficulties to assess correctly the overall effect of a policy measure addressed to one of the two interacting markets, when prices fluctuate due to producers' entry and exit decisions. For instance, the top panels of Fig. 6 represent the effect, on the dynamics of market $X$, of an exogenous change of the supply parameter in market $Z$. More important, simple policy measures could yield unexpected 
effects when combined to the interaction mechanisms described in this paper. This is illustrated in the following example, where we suppose that a central authority tries to support prices in market $Z$ by buying $K_{Z}$ units of commodity $Z$ at each time step. The market clearing equation (1) in market $Z$ would become, in this case, $K_{Z}+D_{Z, t}=N W_{Z, t} S_{Z, t}$, or

$$
\frac{\left(K_{Z} b_{Z}+a_{Z}\right)-P_{Z, t}}{b_{Z}}=N W_{Z, t} S_{Z, t} .
$$

By comparing equation (23) with (1)-(2), we observe that such a strategy is equivalent to an increase in the parameter $a_{Z}$. Fig. 7 (bottom panels) reports bifurcation diagrams of the two prices versus parameter $K_{Z}$. Parameters are as in Figs. 1 and 2 , with $f=0.15$. What can be observed is that the central authority succeeds in supporting price $Z$ (and also price $X$ ). However, if the intervention is too strong, the overall effect may be that of destabilization of the whole system of interacting markets (via Neimark-Sacker bifurcation). It is remarkable that if markets were not connected $(f=0)$, such a measure would simply shift upwards the demand schedule in market $Z$, without changing its slope and therefore preserving the stability properties of the steady state. In contrast, in the case of interdependent cobweb markets, steady state stability may be lost.

\section{Figure 7 goes about here}

Exogenous noise. As is well known, the interaction of nonlinear deterministic dynamical systems with simple exogenous noise processes can considerably enrich the range of possible dynamic scenarios of the models, and give rise to realistic patterns for the time evolution of the relevant economic variables (see e.g. Brock and Hommes 1998, Hommes and Rosser 2001).

The following simple numerical experiment highlights the way exogenous noise interacts with the nonlinear deterministic switching mechanism of our model. Namely, our goal is to assess the impact of noise relative to that of parameter $f$, the latter affecting directly the size and nature of deterministic fluctuations. We introduce i.i.d normally distributed noise in equation (12) of the time evolution of price $Z$, which thus becomes

$$
P_{Z, t}=\frac{a_{Z} d_{Z}-g_{Z}\left(1-\Omega_{t}\right)\left(P_{Z, t-1}-c_{Z}\right)}{d_{Z}}+\sigma_{\epsilon} \widetilde{\epsilon}_{Z}
$$

where $\widetilde{\epsilon}_{Z} \sim \mathcal{N}(0,1)$, and $\sigma_{\epsilon}>0$. This is equivalent to assuming, for instance, a noisy component of the aggregate supply in market $Z{ }^{16}$ In Fig. 8, the top-left panel depicts the behavior of price $X$ versus time, under the same parameter selection of Fig. 1 (beyond the Neimark-Sacker boundary of the stability region) and $\sigma_{\epsilon}=0.2$. The effect of noise seems negligible and doesn't alter qualitatively the deterministic pattern. The price behavior is mainly driven by the underlying deterministic dynamics on a closed orbit (the latter represented in the top panels of Fig. 2). Similar results are observed from experiments with higher $f$ and/or higher $\sigma_{\epsilon}$, that all produce

\footnotetext{
${ }^{16}$ More precisely, if $\sigma_{\xi} \widetilde{\xi}_{Z}, \sigma_{\xi}>0, \widetilde{\xi}_{Z} \sim \mathcal{N}(0,1)$, is the assumed noise on the aggregate supply, $N W_{Z, t} S_{Z, t}$, the resulting noise impacting on price $Z$ turns out to be $-b_{Z} \sigma_{\xi} \widetilde{\xi}_{Z}$, that is, the noise component in equation (24), with $\sigma_{\epsilon}:=b_{Z} \sigma_{\xi}$. Note also that assuming shocks on individual supply functions would be somewhat problematic both in terms of their aggregation and due to their effect on realized profits (and thus on the quantity $\Omega_{t}$ in equation (15)).
} 
price patterns qualitatively similar to the corresponding deterministic cases. To achieve a clearer picture of the effect of the noise on the size of price fluctuations, the top-right panel plots the standard deviation of price $X$ versus the standard deviation $\sigma_{\epsilon}$ of the noise process, and shows that noise has no (or even ambiguous) effects for small $\sigma_{\epsilon}$, whereas the average size of fluctuations tends to increase starting from about $\sigma_{\epsilon}=0.2$. A more clear-cut and much stronger effect can be observed, instead, in the range of parameter $f$ for which the steady state is locally asymptotically stable. An example of a noisy price trajectory is provided in the bottom-left panel, for $f=0.12$ and $\sigma_{\epsilon}=0.2$. Apart from the smaller range of oscillations, the qualitative price pattern with stable underlying deterministic dynamics resembles that of the previous case, where parameter $f$ was outside its stability range $(f=0.17)$. The bottom-right panel represents the joint effect of noise and parameter $f$, in the stability range of the deterministic system. It turns out that for fixed $f$ the standard deviation of price $X$ is approximately proportional to the standard deviation of the noise process impacting on market $Z$. However, a fixed noise level is increasingly amplified by the intensity of choice $f$, so that the standard deviation of price $X$ seems to grow exponentially with $f$. This shows that the combined effect of such exogenous and endogenous sources of price fluctuations may not be trivial.

\section{Figure 8 goes about here}

\section{Conclusions}

Suppose that farmers observe that the price of rye increases. The classical cobweb scenario then predicts that farmers will start to expand their production of rye. However, when the supply becomes too high, the price for rye will decrease again. Farmers will consequently leave the market. Note that cobweb models do not explore what the farmers do when their rye production is low. They will most likely increase their production in another market, say, the wheat market.

The goal of this paper is to investigate such market interactions. To make matters as simple as possible, we stick to the classical cobweb world as far as possible. While the supply function of an individual producer increases linearly in the (expected) price, the total supply is a nonlinear function. The aggregated supply depends on how profitable a market was in the last period. If a market was relatively profitable, it attracts more producers and the total supply increases. Analytical investigation and numerical simulations of our nonlinear model indicate that such market interactions may add to the cyclical behavior of commodity prices captured by the classical cobweb model, and become a further source of instability and complexity. Indeed, from the point of view of the local asymptotic stability of the stationary state, we have proven that the introduction of market interactions can - at least in the symmetric case - destabilize an otherwise stable equilibrium. Moreover, there is a strong connection between the types of local bifurcation of the steady state and the underlying assumptions about risk attitudes / perceptions of producers.

Global inspections have shown that when the agents react more sensitively to profit differentials, interactions become stronger and more complex dynamic scenarios emerge. Market interactions are also quite important from a policy perspective. For example, it is clear from our framework 
(and it has been shown via numerical simulation) that any regulatory intervention that affects the profitability in one market, may have unexpected effects on the markets' relative performance and thus on the whole system of connected commodity markets.

The model proposed in this paper is a very stylized one and could be developed in a number of directions, some of which have been preliminary investigated by means of numerical experiments.

First, the model should be generalized so as to allow demand functions to depend on prices of both goods. This would lead to establish more general results about the steady state and its local stability properties.

Second, a generalization to adaptive expectations schemes seems necessary, in order to assess to what extent the introduction of some 'inertia' in expectation formation can exert a stabilizing effect on price fluctuations. Further generalizations could allow for some heterogeneity across agents in their beliefs about next period prices.

Third, apart from the introduction of time-varying fractions of producers who may switch between two markets, we have followed the classical linear cobweb model as far as possible: a more realistic framework should consider further sources of nonlinearity - following recent literature on cobweb models - e.g. nonlinear demand curves, time-varying second moment beliefs, etc.

Fourth, the analysis of the global effect of various kinds of regulatory interventions within our simple framework of interacting markets would be an interesting and challenging task.

\section{References}

Baak, S., 1999. Test for bounded rationality with a linear dynamic model distorted by heterogeneous expectations. Journal of Economic Dynamics and Control 23, 1517-1543.

Borenzstein, E., Kann, M., Reinhart, C., Wickham, P., 1994. The behavior of nonoil commodity prices. IMF Occasional Paper 112. Washington: IMF.

Boussard, J.-M., 1996. When risk generates chaos. Journal of Economic Behavior and Organization 29, 433-446.

Branch, W., 2002. Local convergence properties of a cobweb model with rationally heterogeneous expectations. Journal of Economic Dynamics and Control 27, 63-85.

Brock, W.A., Hommes, C.H., 1997. A rational route to randomness. Econometrica 65, 10591095.

Brock, W.A., Hommes, C.H., 1998. Heterogeneous beliefs and routes to chaos in a simple asset pricing model. Journal of Economic Dynamics and Control 22, 1235-1274.

Cashin, P., McDermott, J., Scott, A., 2002. Booms and slumps in world commodity prices. Journal of Development Economics 69, 277-296.

Chavas, J., 2000. On information and market dynamics: The case of the U.S. beef market. Journal of Economic Dynamics and Control 24, 833-853.

Chiarella, C., 1988. The cobweb model, its instability and the onset of chaos. Economic Modelling 5, 377-384.

Chiarella, C., He, X.-Z., 2003. Dynamics of beliefs and learning under $\mathbf{a}_{L^{-}}$processes - the heterogeneous case. Journal of Economic Dynamics and Control 27, 503-532. 
Chiarella, C., He, X.-Z., Hung, H., Zhu, P., 2006. An analysis of the cobweb model with bounded rational heterogeneous producers. Journal of Economic Behavior and Organization 61, 750-768.

Currie, M., Kubin, I., 1995. Non-linearities and partial analysis. Economics Letters 49, 27-31.

Day, R.H., 1994. Complex economic dynamics: An introduction to dynamical systems and market mechanisms. Cambridge, MA: MIT Press.

Deaton, A., 1999. Commodity prices and growth in Africa. Journal of Economic Perspectives 13, 23-40.

Dieci, R., Westerhoff, F., 2009. Stability analysis of a cobweb model with market interactions. Applied Mathematics and Computation 215, 2011-2023.

Ezekiel, M., 1938. The cobweb theorem. Quarterly Journal of Economics 52, 255-280.

Goeree, J.K., Hommes, C.H., 2000. Heterogeneous beliefs and the non-linear cobweb model. Journal of Economic Dynamics and Control 24, 761-798.

Hommes, C.H., 1994. Dynamics of the cobweb model with adaptive expectations and non-linear supply and demand. Journal of Economic Behavior and Organization 24, 315-335.

Hommes, C.H., 1998. On the consistency of backward-looking expectations: The case of the cobweb. Journal of Economic Behavior and Organization 33, 333-362.

Hommes, C.H., van Eekelen, A., 1996. Partial equilibrium analysis in a noisy chaotic market. Economics Letters 53, 275-282.

Hommes, C.H., Rosser, J.B., Jr., 2001. Consistent expectations equilibria and complex dynamics in renewable resource markets. Macroeconomic Dynamics 5, 180-203.

Hommes, C.H., Sonnemans, J., Tuinstra, J., van de Velden, H., 2007. Learning in cobweb experiments. Macroeconomic Dynamics 11, 8-33.

Lasselle, L., Svizzero, S., Tidsell, C., 2005. Stability and cycles in a cobweb model with heterogeneous expectations. Macroeconomic Dynamics 9, 630-650.

Medio, A., Lines, M., 2001. Nonlinear Dynamics. A primer. Cambridge: Cambridge University Press.

Nerlove, M., 1958. Adaptive expectations and cobweb phenomena. Quarterly Journal of Economics 73, 227-240.

Onozaki, T., Sieg, G., Yokoo, M., 2003. Stability, chaos and multiple attractors: a single agent makes a difference. Journal of Economic Dynamics and Control 27, 1917-1938.

Sonnemans, J., Hommes, C.H., Tuinstra, J., van de Velden, H., 2004. The instability of a heterogeneous cobweb economy: A strategy experiment on expectation formation. Journal of Economic Behavior and Organization 54, 453-481.

Yousefi, S., Maistrenko, Y., Popovych, S., 2000. Complex dynamics in a simple model of interdependent open economies. Discrete Dynamics in Nature and Society 5, 161-177.

\section{Appendix A1. Derivation of the supply curve for risk-averse producers}

In this Appendix we show how the producer's supply curve can be derived within a standard one-period mean-variance framework (see also Boussard 1996, Chiarella et al. 2006). The same 
framework allows the derivation of a relationship between parameters $d_{X}$ and $e_{X}, d_{Z}$ and $e_{Z}$. In the following, we remove subscripts $X$ and $Z$ in order to simplify the notation.

Consider a single risk-averse producer with exponential utility function

$$
u(x)=-\exp (-\gamma x)
$$

where $\gamma>0$ is the (constant) absolute risk aversion. In period $t-1$ the producer forms his/her beliefs about the expectation and the variance of the price in period $t$, which we denote by $E_{t-1}\left[P_{t}\right]$ and $\operatorname{Var}_{t-1}\left[P_{t}\right]$, respectively. Assume, for simplicity, naïve expectations and constant secondmoment beliefs, $E_{t-1}\left[P_{t}\right]=P_{t-1}, \operatorname{Var}_{t-1}\left[P_{t}\right]=\sigma_{P}^{2}$. Assume also a quadratic cost function, $C(S)=$ $c S+e S^{2}$, where $S$ denotes the quantity to be supplied in period $t$. For a given output decision $S_{t}$, the random profit in period $t$ is given by

$$
\pi_{t}=P_{t} S_{t}-C\left(S_{t}\right)=\left(P_{t}-c\right) S_{t}-e S_{t}^{2}
$$

while conditional expectation and variance of the profit are given by

$$
E_{t-1}\left[\pi_{t}\right]=\left(P_{t-1}-c\right) S_{t}-e S_{t}^{2}
$$

and

$$
\operatorname{Var}_{t-1}\left[\pi_{t}\right]=S_{t}^{2} \sigma_{P}^{2}
$$

respectively.

Then the risk-averse producer solves ${ }^{17}$

$$
\max _{S_{t}} E_{t-1}\left[u\left(\pi_{t}\right)\right]
$$

Under the utility function (25) and other standard assumptions (conditional normality of the profit in agent's beliefs ${ }^{18}$ ), the problem (28) can be reformulated as:

$$
\max _{S_{t}}\left\{E_{t-1}\left[\pi_{t}\right]-\frac{\gamma}{2} \operatorname{Var}_{t-1}\left[\pi_{t}\right]\right\}
$$

i.e. using (26) and (27):

$$
\max _{S_{t}}\left\{\left(P_{t-1}-c\right) S_{t}-\left(e+\frac{\gamma}{2} \sigma_{P}^{2}\right) S_{t}^{2}\right\}
$$

By setting

$$
d=2\left(e+\frac{\gamma}{2} \sigma_{P}^{2}\right)
$$

\footnotetext{
${ }^{17}$ Under the assumed utility function, the problem (28) is obviously equivalent to maximising expected utility of wealth, $E_{t-1}\left[u\left(Y_{t}\right)\right]$, where $Y_{t}$ denotes producer's random wealth at time $t$, defined recursively as $Y_{t}=Y_{t-1}+\pi_{t}$.

${ }^{18}$ In order to stick to the mean-variance framework (which preserves analytical tractability) we assume that producers treat the price as a random variable drawn from a normal distribution, whose mean and variance they are seeking to learn.
} 
the solution of the expected utility maximization problem (29) becomes

$$
S_{t}=\frac{P_{t-1}-c}{d}
$$

where $d>2 e$ because both $\gamma$ and $\sigma_{P}^{2}$ are positive in (30). Note that the extreme cases of risk neutrality, $\gamma \rightarrow 0$, or zero (perceived) risk, $\sigma_{P}^{2} \rightarrow 0$, imply that $d \rightarrow 2 e$.

\section{Appendix A2. Existence and uniqueness of the steady state}

The steady states are obtained by setting $\left(P_{X, t}, P_{Z, t}, S_{X, t}, S_{Z, t}\right)=\left(P_{X, t-1}, P_{Z, t-1}, S_{X, t-1}, S_{Z, t-1}\right)=$ $\left(\bar{P}_{X}, \bar{P}_{Z}, \bar{S}_{X}, \bar{S}_{Z}\right)$ in the dynamical system (11)-(14). This leads to the following system of equations

$$
\begin{gathered}
\bar{P}_{X}=\frac{a_{X} d_{X}+g_{X}(1+\bar{\Omega})\left(c_{X}-\bar{P}_{X}\right)}{d_{X}}, \quad \bar{P}_{Z}=\frac{a_{Z} d_{Z}+g_{Z}(1-\bar{\Omega})\left(c_{Z}-\bar{P}_{Z}\right)}{d_{Z}}, \\
\bar{S}_{X}=\frac{\bar{P}_{X}-c_{X}}{d_{X}}, \quad \bar{S}_{Z}=\frac{\bar{P}_{Z}-c_{Z}}{d_{Z}}
\end{gathered}
$$

where $\bar{\Omega}$ is given by

$$
\bar{\Omega}=\tanh \left\{\frac{f}{2}\left[\left(\bar{P}_{X}-c_{X}\right) \bar{S}_{X}-e_{X} \bar{S}_{X}^{2}-\left(\bar{P}_{Z}-c_{Z}\right) \bar{S}_{Z}+e_{Z} \bar{S}_{Z}^{2}\right]\right\}
$$

and where $\left(\bar{P}_{X}-c_{X}\right) \bar{S}_{X}-e_{X} \bar{S}_{X}^{2}:=\bar{\pi}_{X}$ and $\left(\bar{P}_{Z}-c_{Z}\right) \bar{S}_{Z}-e_{Z} \bar{S}_{Z}^{2}:=\bar{\pi}_{Z}$ represent the steady state profits.

First, note that from (31)-(32), $\bar{P}_{X}, \bar{P}_{Z}, \bar{S}_{X}$, and $\bar{S}_{Z}$ can be expressed as functions of $\bar{\Omega}$, yielding (17) and (18), i.e.

$$
\begin{gathered}
\bar{P}_{X}=\frac{a_{X} d_{X}+g_{X}(1+\bar{\Omega}) c_{X}}{d_{X}+g_{X}(1+\bar{\Omega})}, \quad \bar{P}_{Z}=\frac{a_{Z} d_{Z}+g_{Z}(1-\bar{\Omega}) c_{Z}}{d_{Z}+g_{Z}(1-\bar{\Omega})}, \\
\bar{S}_{X}=\frac{a_{X}-c_{X}}{d_{X}+g_{X}(1+\bar{\Omega})}, \quad \bar{S}_{Z}=\frac{a_{Z}-c_{Z}}{d_{Z}+g_{Z}(1-\bar{\Omega})} .
\end{gathered}
$$

Second, note that for both market $X$ and market $Z$ (we omit the subscripts for simplicity) the following relations hold:

$$
(\bar{P}-c) \bar{S}=\frac{(\bar{P}-c)^{2}}{d}, \quad e \bar{S}^{2}=e \frac{(\bar{P}-c)^{2}}{d^{2}}=\frac{e}{d} \frac{(\bar{P}-c)^{2}}{d},
$$

and therefore we obtain for the steady-state profit

$$
\bar{\pi}=(\bar{P}-c) \bar{S}-e \bar{S}^{2}=\frac{d-e}{d} \frac{(\bar{P}-c)^{2}}{d}=(d-e) \bar{S}^{2}
$$

Substituting into (33), one obtains nonlinear equation (16), i.e.

$$
\Omega=\tanh \left\{\frac{f}{2}\left[\frac{\left(d_{X}-e_{X}\right)\left(a_{X}-c_{X}\right)^{2}}{\left[d_{X}+g_{X}(1+\Omega)\right]^{2}}-\frac{\left(d_{Z}-e_{Z}\right)\left(a_{Z}-c_{Z}\right)^{2}}{\left[d_{Z}+g_{Z}(1-\Omega)\right]^{2}}\right]\right\},
$$


that implicitly defines the steady-state distribution of producers across markets, $\bar{\Omega}$. It is easy to prove that equation (16) admits a unique solution for $\Omega$ ranging in $[-1,1]$. Indeed, under our assumptions about the parameters $\left(a_{X}>c_{X}, a_{Z}>c_{Z}, d_{X}>e_{X}, d_{Z}>e_{Z}\right)$, the righthand side of (16) is a strictly decreasing function of $\Omega$ - let us denote it by $Q(\Omega)$ - and therefore $-1<Q(1)<Q(-1)<1$, due to the fact that $-1<\tanh (x)<1$ for any $x$. It follows that a solution to the equation $\Omega=Q(\Omega), \Omega \in[-1,1]$, exists and that it is unique. Denoting this solution by $\bar{\Omega}$, the equilibrium prices and quantities $\bar{P}_{X}, \bar{P}_{Z}, \bar{S}_{X}, \bar{S}_{Z}$ are thus given by (17) and (18). Note that the steady state cannot be explicitly computed in general, due to the fact that (16) cannot be solved analytically.

\section{Appendix A3. Local stability conditions in the symmetric case}

In the symmetric case, the time evolution of the dynamical system is driven by the iteration of the following nonlinear map (where the symbol ' denotes the unit time advancement operator)

$$
T:\left\{\begin{array}{l}
P_{X}^{\prime}=F_{1}\left(P_{X}, P_{Z}, S_{X}, S_{Z}\right)=a-\frac{g}{d}(1+\Omega)\left(P_{X}-c\right) \\
P_{Z}^{\prime}=F_{2}\left(P_{X}, P_{Z}, S_{X}, S_{Z}\right)=a-\frac{g}{d}(1-\Omega)\left(P_{Z}-c\right) \\
S_{X}^{\prime}=G_{1}\left(P_{X}\right)=\frac{1}{d}\left(P_{X}-c\right) \\
S_{Z}^{\prime}=G_{2}\left(P_{Z}\right)=\frac{1}{d}\left(P_{Z}-c\right)
\end{array}\right.
$$

where

$$
\Omega=\tanh \left[\frac{f}{2}\left(\pi_{X}-\pi_{Z}\right)\right], \quad \pi_{X}=\left(P_{X}-c\right) S_{X}-e S_{X}^{2}, \quad \pi_{Z}=\left(P_{Z}-c\right) S_{Z}-e S_{Z}^{2}
$$

In order to compute the Jacobian matrix we first determine the partial derivatives of $\Omega=\Omega\left(P_{X}, P_{Z}, S_{X}, S_{Z}\right)$ with respect to the state variables. We obtain

$$
\begin{gathered}
\frac{\partial \Omega}{\partial P_{X}}=\left\{1-\tanh ^{2}\left[\frac{f}{2}\left(\pi_{X}-\pi_{Z}\right)\right]\right\} \frac{f}{2} S_{X} \\
\frac{\partial \Omega}{\partial P_{Z}}=\left\{1-\tanh ^{2}\left[\frac{f}{2}\left(\pi_{X}-\pi_{Z}\right)\right]\right\}\left(-\frac{f}{2} S_{Z}\right), \\
\frac{\partial \Omega}{\partial S_{X}}=\left\{1-\tanh ^{2}\left[\frac{f}{2}\left(\pi_{X}-\pi_{Z}\right)\right]\right\} \frac{f}{2}\left[\left(P_{X}-c\right)-2 e S_{X}\right], \\
\frac{\partial \Omega}{\partial S_{Z}}=\left\{1-\tanh ^{2}\left[\frac{f}{2}\left(\pi_{X}-\pi_{Z}\right)\right]\right\} \frac{f}{2}\left[-\left(P_{Z}-c\right)+2 e S_{Z}\right] .
\end{gathered}
$$

The partial derivatives of $F_{1}$ and $F_{2}$ are the following

$$
\begin{gathered}
\frac{\partial F_{1}}{\partial P_{X}}=-\frac{g}{d}\left[\frac{\partial \Omega}{\partial P_{X}}\left(P_{X}-c\right)+(1+\Omega)\right] \\
\frac{\partial F_{1}}{\partial P_{Z}}=-\frac{g}{d} \frac{\partial \Omega}{\partial P_{Z}}\left(P_{X}-c\right), \quad \frac{\partial F_{1}}{\partial S_{X}}=-\frac{g}{d} \frac{\partial \Omega}{\partial S_{X}}\left(P_{X}-c\right), \quad \frac{\partial F_{1}}{\partial S_{Z}}=-\frac{g}{d} \frac{\partial \Omega}{\partial S_{Z}}\left(P_{X}-c\right),
\end{gathered}
$$




$$
\begin{gathered}
\frac{\partial F_{2}}{\partial P_{Z}}=\frac{g}{d}\left[\frac{\partial \Omega}{\partial P_{Z}}\left(P_{Z}-c\right)-(1-\Omega)\right] \\
\frac{\partial F_{2}}{\partial P_{X}}=\frac{g}{d} \frac{\partial \Omega}{\partial P_{X}}\left(P_{Z}-c\right), \quad \frac{\partial F_{2}}{\partial S_{X}}=\frac{g}{d} \frac{\partial \Omega}{\partial S_{X}}\left(P_{Z}-c\right), \quad \frac{\partial F_{2}}{\partial S_{Z}}=\frac{g}{d} \frac{\partial \Omega}{\partial S_{Z}}\left(P_{Z}-c\right),
\end{gathered}
$$

while the derivatives of $G_{1}$ and $G_{2}$ are

$$
\frac{d G_{1}}{d P_{X}}=\frac{d G_{2}}{d P_{Z}}=\frac{1}{d}
$$

In order to evaluate the Jacobian at the steady state $\mathbf{q}^{*}=(\bar{P}, \bar{P}, \bar{S}, \bar{S})$, where $\bar{P}=(a d+c g) /(d+g)$, $\bar{S}=(\bar{P}-c) / d=(a-c) /(d+g)$, we note that the steady-state profits can be rewritten as

$$
\bar{\pi}_{X}=\bar{\pi}_{Z}=(d-e) \bar{S}^{2}=(d-e) \frac{(a-c)^{2}}{(d+g)^{2}}
$$

The Jacobian matrix is thus given by

$$
D T\left(\mathbf{q}^{*}\right)=\left[\begin{array}{cccc}
-g \frac{f}{2} \bar{S}^{2}-\frac{g}{d} & g \frac{f}{2} \bar{S}^{2} & -g \frac{f}{2}(d-2 e) \bar{S}^{2} & g \frac{f}{2}(d-2 e) \bar{S}^{2} \\
g \frac{f}{2} \bar{S}^{2} & -g \frac{f}{2} \bar{S}^{2}-\frac{g}{d} & g \frac{f}{2}(d-2 e) \bar{S}^{2} & -g \frac{f}{2}(d-2 e) \bar{S}^{2} \\
\frac{1}{d} & 0 & 0 & 0 \\
0 & \frac{1}{d} & 0 & 0
\end{array}\right],
$$

Tedious computations allow the factorization of the characteristic polynomial of $D T\left(\mathbf{q}^{*}\right)$ as follows

$$
\begin{aligned}
& P(\lambda)=\lambda\left(\frac{g}{d}+\lambda\right)\left[\lambda^{2}+\left(g f \bar{S}^{2}+\frac{g}{d}\right) \lambda+\frac{g f(d-2 e) \bar{S}^{2}}{d}\right]= \\
& \lambda\left(\frac{g}{d}+\lambda\right) P_{1}(\lambda) .
\end{aligned}
$$

It follows that two eigenvalues of $D T\left(\mathbf{q}^{*}\right)$, say $\lambda_{1}$ and $\lambda_{2}$, are $\lambda_{1}=0, \lambda_{2}=-g / d$, respectively, while the remaining two eigenvalues, say $\lambda_{3}$ and $\lambda_{4}$, are the roots of

$$
P_{1}(\lambda):=\lambda^{2}+\left(g f \bar{S}^{2}+\frac{g}{d}\right) \lambda+\frac{g f(d-2 e) \bar{S}^{2}}{d} .
$$

Hence a sufficient condition for the steady state $\mathbf{q}^{*}$ to be locally asymptotically stable is provided by the following set of inequalities ${ }^{19}$

$$
\begin{aligned}
\frac{g}{d} & <1, \\
P_{1}(1)>0, \quad P_{1}(-1) & >0, \quad P_{1}(0)<1 .
\end{aligned}
$$

In particular, conditions (35) give a necessary and sufficient condition for the eigenvalues $\lambda_{3}$ and $\lambda_{4}$ to be of modulus smaller than unity (see, e.g. Medio and Lines 2001). Condition $P_{1}(1)>0$ is always true under our assumptions about the parameters; condition $P_{1}(-1)>0$ can be rewritten

\footnotetext{
${ }^{19}$ We recall that $g$ and $d$ are positive parameters.
} 
as

$$
\frac{g}{d}<\frac{1}{1+2 e f \bar{S}^{2}}:=u_{F}
$$

while condition $P_{1}(0)<1$ becomes

$$
\frac{g}{d}<\frac{1}{f(d-2 e) \bar{S}^{2}}:=u_{N S}
$$

Note first that $u_{F}<1$, provided that $f>0$, which implies that condition (34) is redundant. This means that - at least for the symmetric case at hand - the stability conditions in the case of interacting markets are stricter than the stability condition (21), which holds in the case of two independent markets. The stability conditions (34)-(35) are thus equivalent to the following

$$
\frac{g}{d}<\min \left\{u_{F}, u_{N S}\right\}
$$

Furthermore, if it happens that $g / d=u_{F}<u_{N S}$, then one of the two eigenvalues $\lambda_{3}$ and $\lambda_{4}$ is equal to -1 (whereas the other still is smaller than one in modulus) and stability is lost via Flip-bifurcation if $g / d$ becomes larger than $u_{F}$ under a small parameter change. On the other hand, when $g / d=u_{N S}<u_{F}$, then $\lambda_{3}$ and $\lambda_{4}$ are complex conjugate with modulus equal to one, and stability is lost via Neimark-Sacker bifurcation if $g / d$ becomes larger than $u_{N S}$. Note also that in the extreme case $f \rightarrow 0$, markets are no longer interdependent and the stability conditions reduce to $g / d<1$, as one would expect. Moreover, in the extreme case $d \rightarrow 2 e$, condition (37) is fulfilled for any selection of parameters, which means that a Neimark-Sacker bifurcation cannot occur in this case.

\section{Figure captions}

\section{Figure 1}

Interacting cobweb markets. The panels show from top to bottom the price in market $X$, the profitability of market $X$, the price in market $Z$, the profitability of market $Z$ and the distribution of producers. Parameter setting is $a=20, b=6, c=2, d=8, e=1, f=0.17$, characterized by quasiperiodic motion on an attracting closed curve in the phase space, born via Neimark-Sacker bifurcation.

\section{Figure 2}

Phase space plots. The panels show the dynamics in phase space, after omitting a transient phase, of the case of symmetric markets for $a=20, b=6, c=2, d=8, e=1, f=0.17$ (top panels), $f=0.20$ (bottom panels). The motion takes place on an invariant closed curve in the phase space. The left panels represent projections on the plane $P_{X}, P_{Z}$, the right panels show projections on the plane $P_{X}, S_{X}$.

Figure 3 
Coexistence of attractors in the symmetric case, for $a=20, b=6, c=2, d=8, e=1$, $f=0.64295$. The two top panels represent the dynamics of $P_{X}$ versus time, after omitting a long transient phase, in the case of two different initial conditions, both selected very close to the repelling steady state, but belonging to different basins of attraction: one trajectory converges to a strange attractor (first panel, initial condition $P_{X, 0}=\bar{P}-0.1, P_{Z, 0}=\bar{P}+0.1, S_{X, 0}=S_{Z, 0}=\bar{S}$ ), the other to an attracting 2-cycle (second panel, initial condition $P_{X, 0}=\bar{P}-0.05, P_{Z, 0}=\bar{P}+0.05$, $S_{X, 0}=S_{Z, 0}=\bar{S}$ ). The third line of panels represents the projections of the coexisting attractors on the plane $P_{X}, P_{Z}$. The two basins of attraction, along the two-dimensional section of equation $S_{X}=S_{Z}=\bar{S}$, are represented with black (period-2 orbit) and white (competing attractor) in the bottom panel.

\section{Figure 4}

Bifurcation diagrams in the case of symmetric markets. The base parameter set is $a=20$, $b=6, c=2, d=8, e=1, f=0.17$. The panels show from top to bottom, bifurcation diagrams for parameters $d, b, c$ and $f$, respectively. These parameters are increased in 600 discrete steps, as indicated on the axis.

\section{Figure 5}

Two-parameter bifurcation diagram for $d_{Z}$ and $f$, showing the existence of two kinds of local bifurcations also in the case of asymmetric markets. The remaining parameters are $a_{X}=20$, $b_{X}=6, c_{X}=2, d_{X}=8, e_{X}=1, a_{Z}=18, b_{Z}=4, c_{Z}=1, e_{Z}=2$. The two smaller panels, representing projections of the attractors in the plane $P_{X}, S_{X}$, correspond to parameter combinations outside the stability region, with $d_{Z}=6, f=0.2$ (two-cycle), and $d_{Z}=8, f=0.24$ (closed curve), respectively.

\section{Figure 6}

Asymmetric markets. Effect of perturbations of the symmetric case, obtained by varying parameters of market $Z$. Apart from those parameters that are varied from time to time, we adopt our base selection for the symmetric case (top panels of Fig. 2), i.e. $a_{X}=a_{Z}=20, b_{X}=b_{Z}=6$, $c_{X}=c_{Z}=2, e_{X}=e_{Z}=1, d_{X}=d_{Z}=8, f=0.17$. The first two lines of panels consider the effect of decreasing supply parameter $d_{Z}$ in market $Z$. The panels of the first line are phase plots in the plane $P_{X}, S_{X}$, for $d_{X}=d_{Z}=8$ and $d_{X}=8, d_{Z}=6$, respectively. The panels of the second line represent the corresponding trajectories in the time domain. The panels of the third line summarize in bifurcation diagrams the asymptotic behavior of prices $P_{X}$ and $P_{Z}$ versus parameter $d_{Z}$. The same is done in the two bottom lines of panels for parameters $b_{Z}$ and $c_{Z}$, respectively.

\section{Figure 7}

Deterministic extensions. Top panel: A bifurcation diagram showing the effect on price $X$ of the introduction of cross-elasticities in demand functions. Case $h=0$ corresponds to our model with the same parameter selection of Fig. 1. For positive $h$ the two goods are complements, for negative $h$ they are substitutes. Central and bottom panels: Bifurcation diagrams showing the effect on prices $X$ and $Z$ of an exogenous demand component $K_{Z}$ for commodity $Z$ by a central authority. Parameters are as in Figs. 1 and 2, except for $f=0.15$. 


\section{Figure 8}

Effect on price $X$ of exogenous i.i.d. normally distributed noise impacting on market $Z$. Top-left panel: A noisy trajectory of price $X$ with parameters as in Fig. 1 and standard deviation of noise on price $Z \sigma_{\epsilon}=0.2$. Top-right panel: Standard deviation of price $X$ versus standard deviation of noise on price $Z$. Bottom-left panel: A noisy trajectory of price $X$, with $\sigma_{\epsilon}=0.2$ and parameters as in the top-left panel, except for $f=0.12$. Bottom-right panel: Standard deviation of price $X$ as

a function of various combinations of parameter $f$ and standard deviation $\sigma_{\epsilon}$. Parameters are as in the previous panels, with $\sigma_{\epsilon}$ ranging from 0 to 0.5 and $f$ ranging from 0 to 0.16 . 

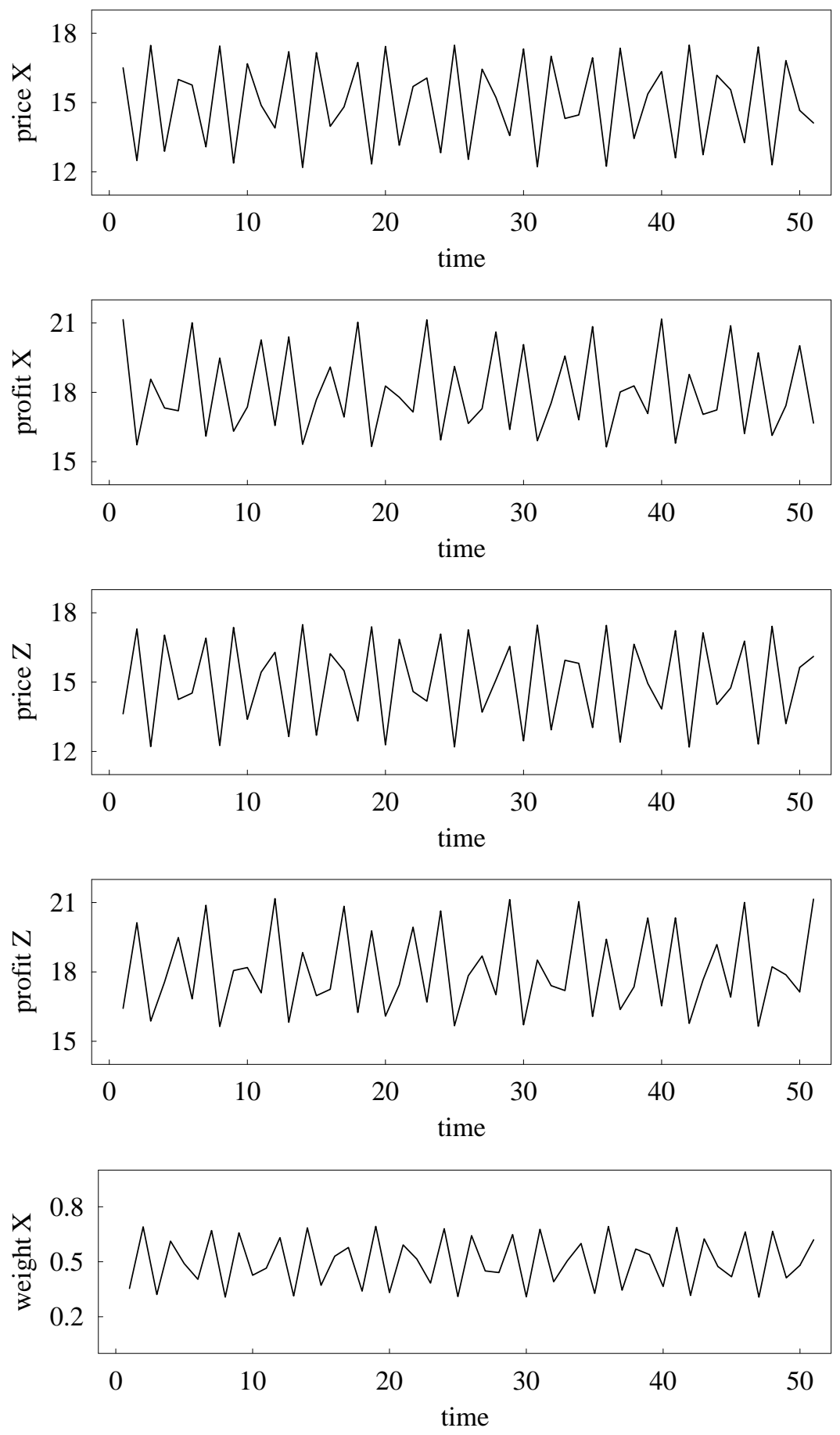

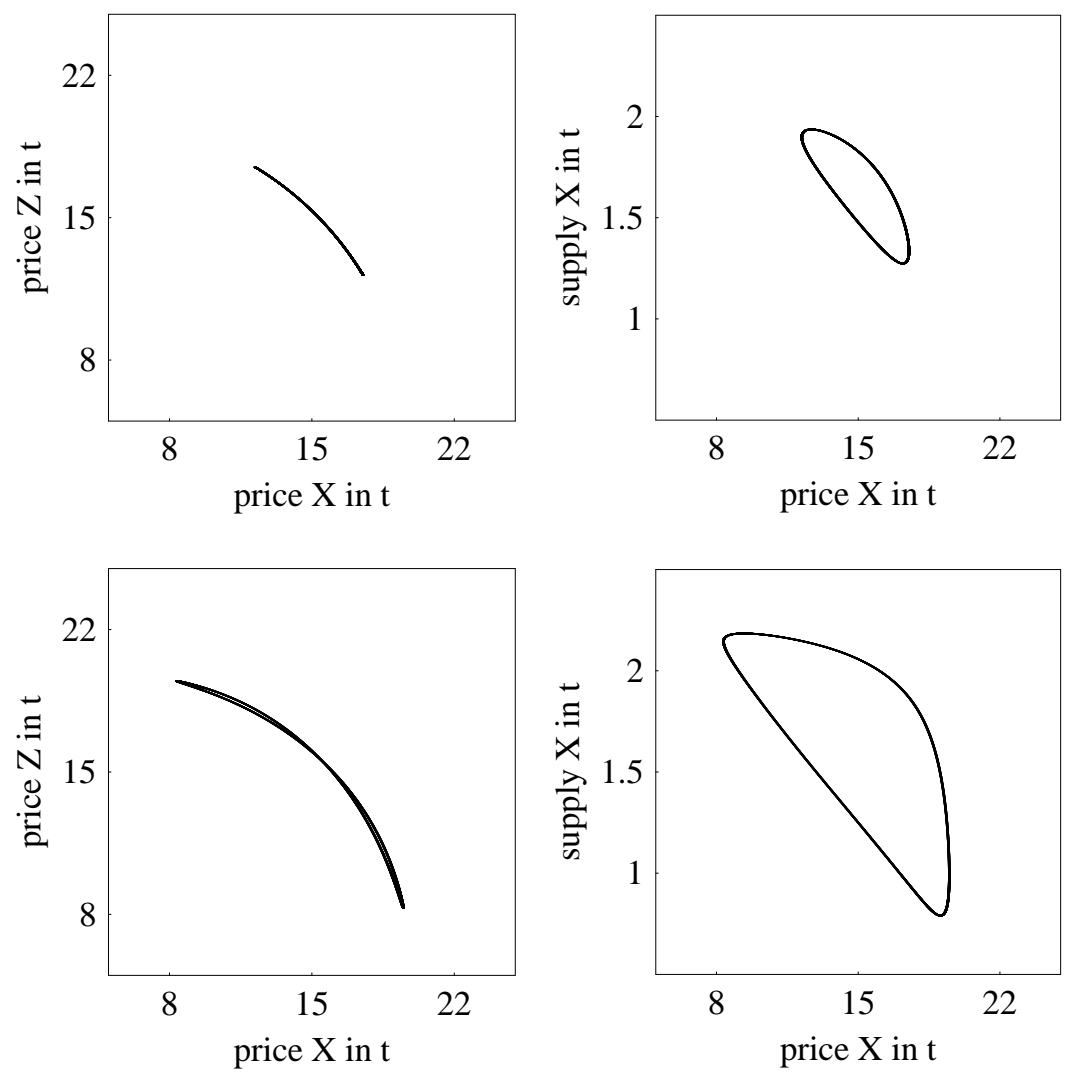


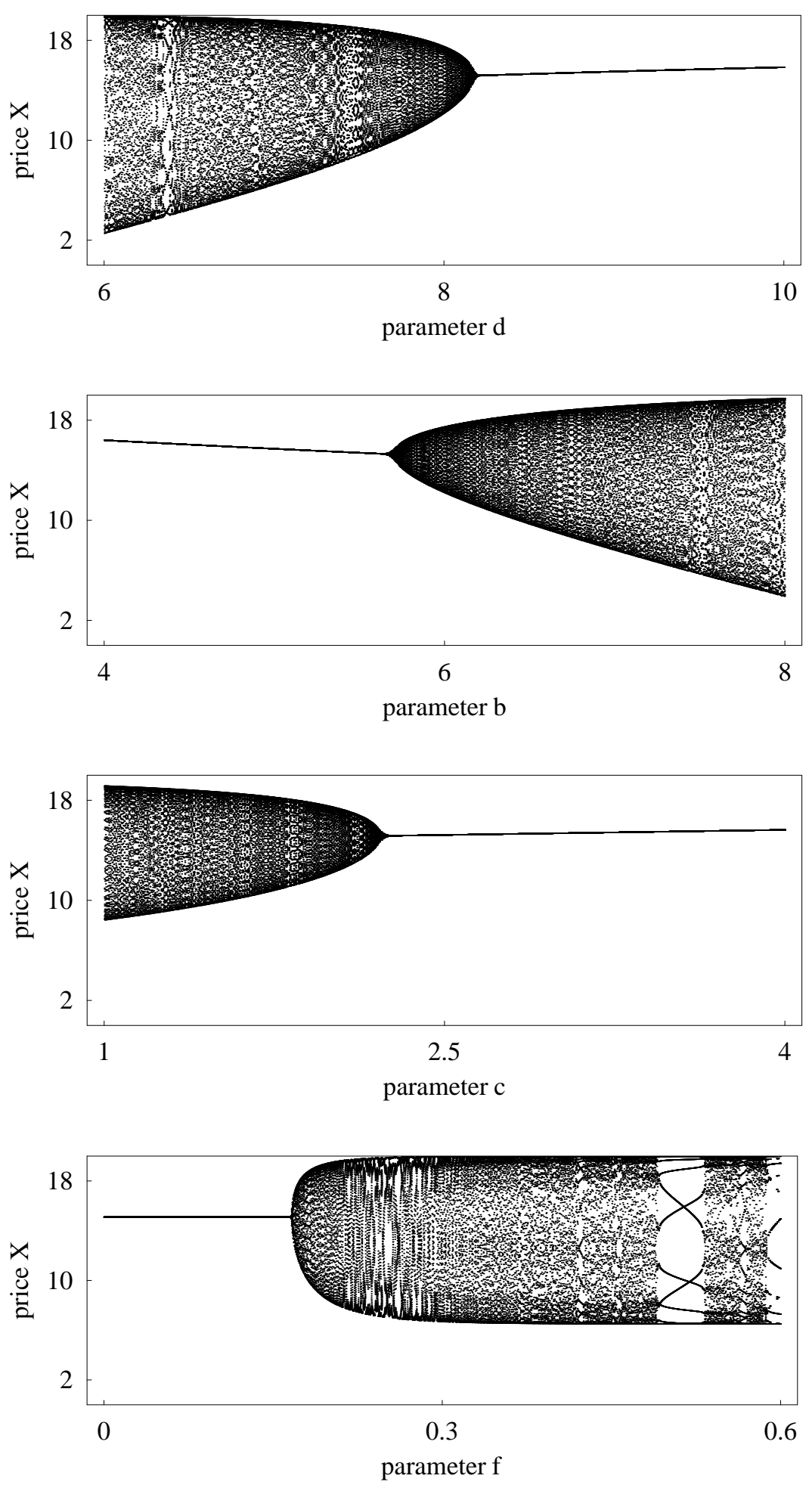

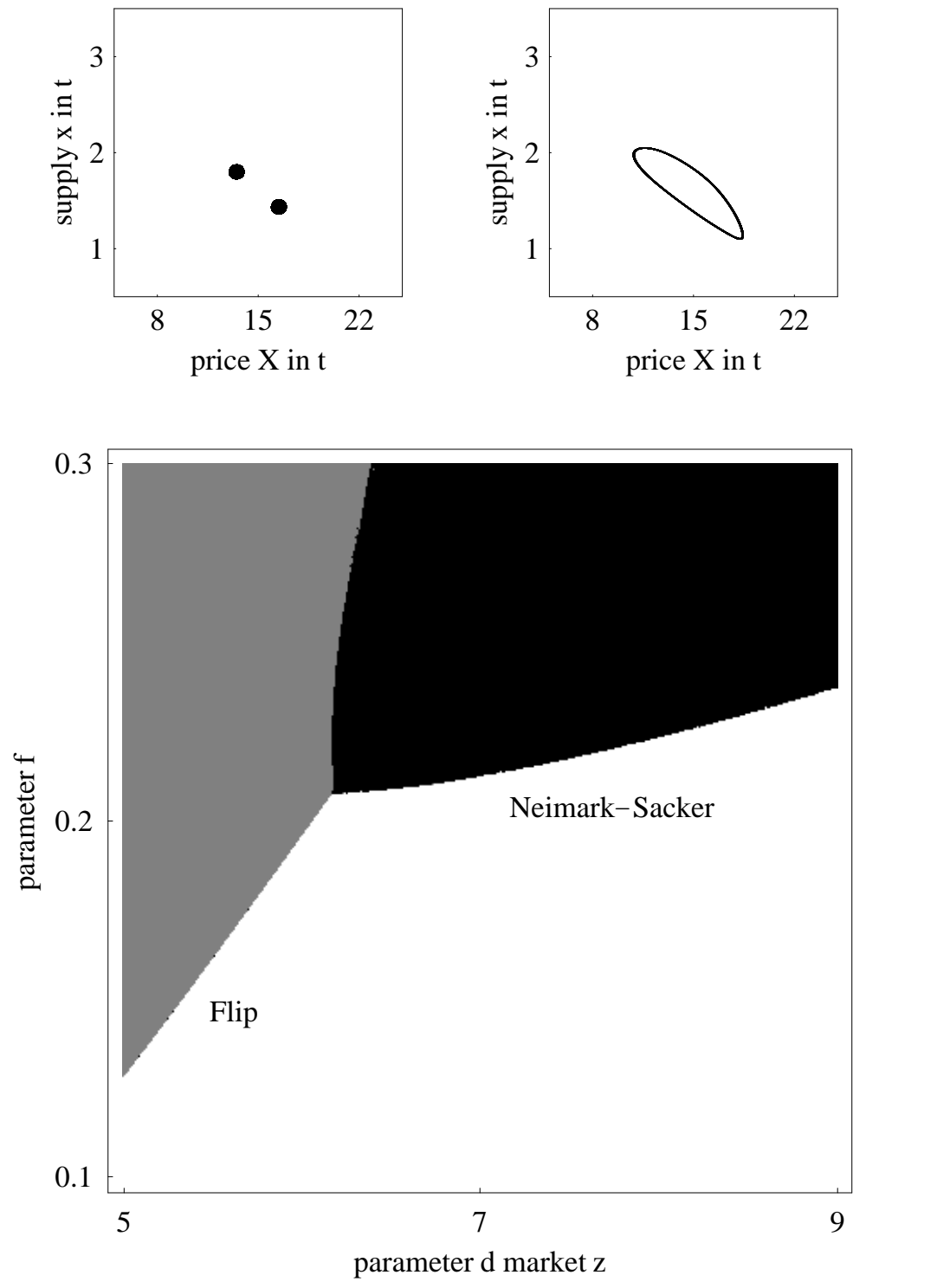

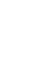

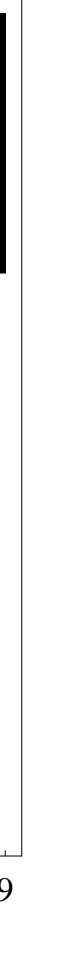

price $\mathrm{X}$ in $\mathrm{t}$

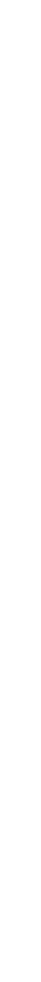



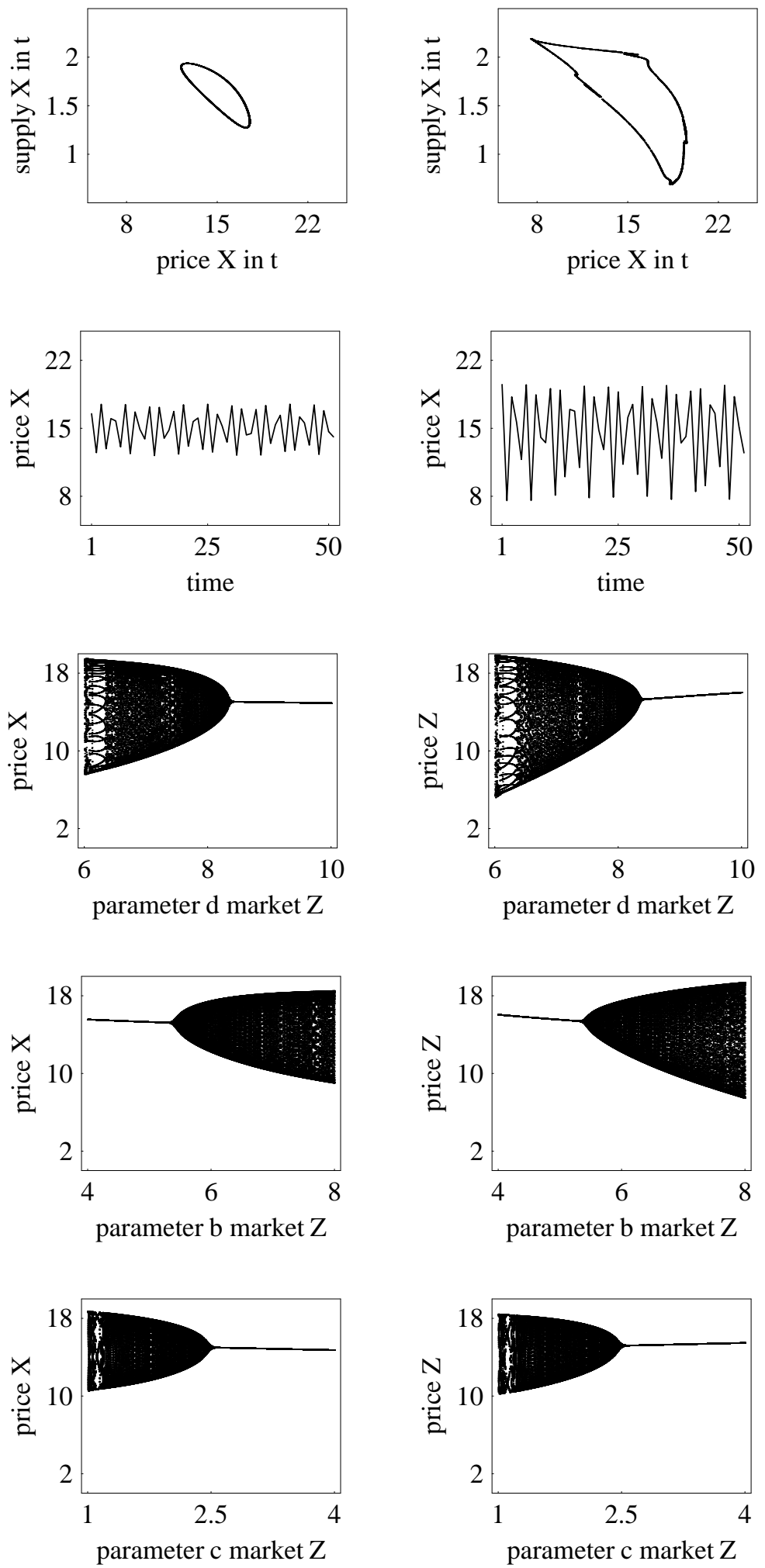

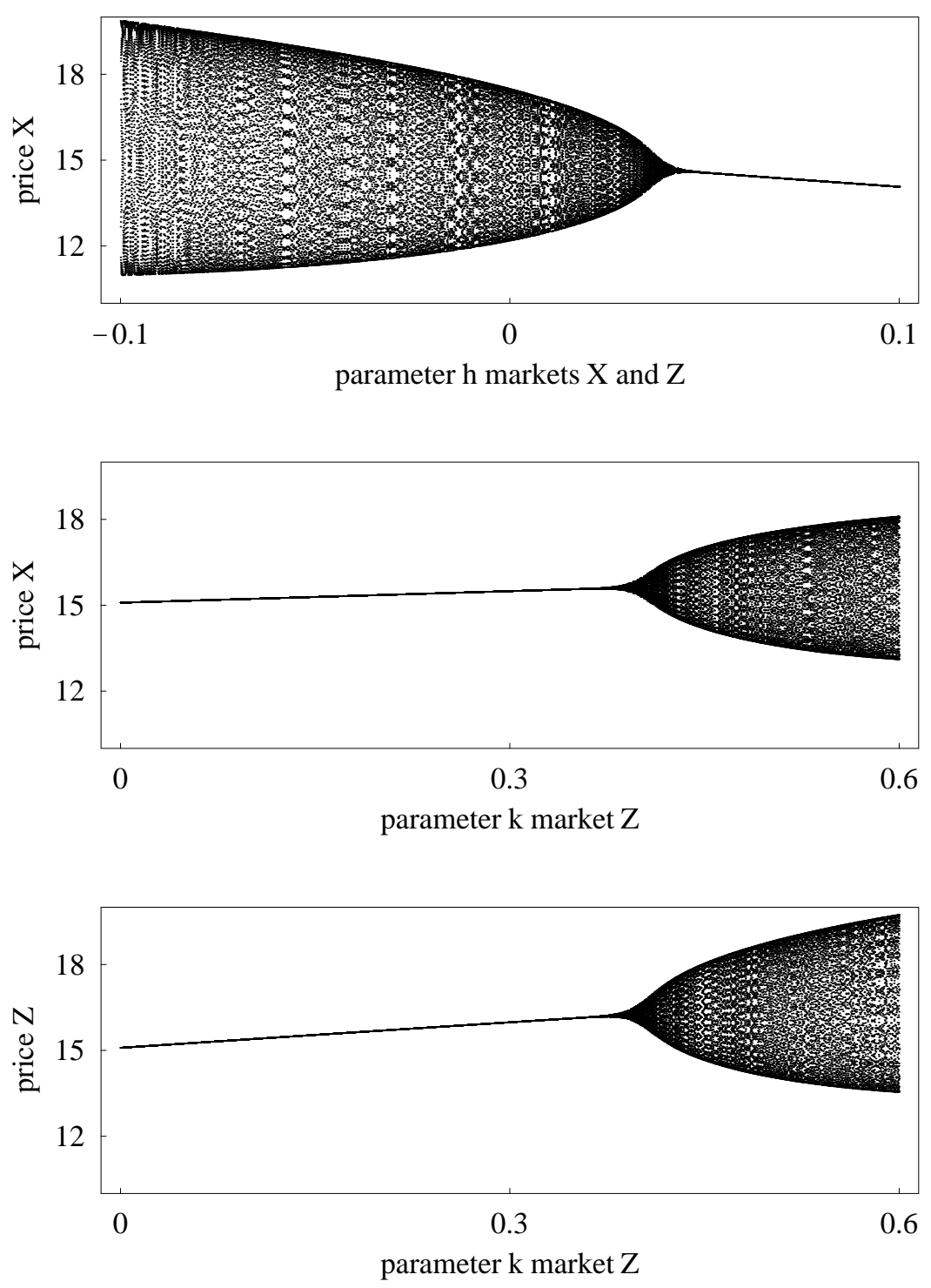

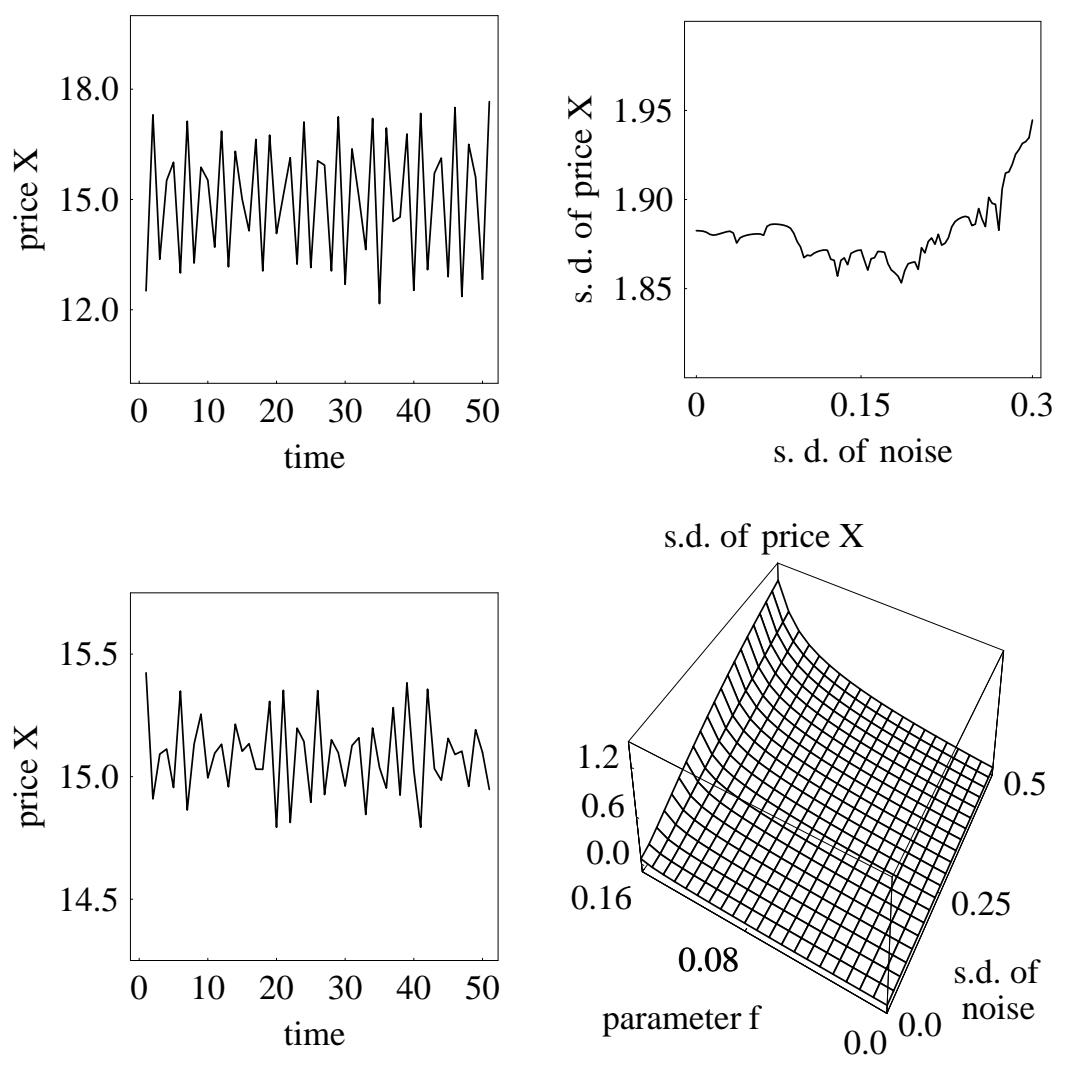